\title{
Gamma irradiation as a practical alternative to preserve the chemical and bioactive wholesomeness of widely used aromatic plants
}

\author{
Eliana Pereira a , Amilcar L. Antonio a,b João C.M. Barreira ${ }^{\mathrm{a}, \mathrm{c}}$, Lillian Barros ${ }^{\mathrm{a}}$, \\ Albino Bento ${ }^{a}$, Isabel C.F.R. Ferreira ${ }^{a, *}$ \\ a Centro de Investigação de Montanha (CIMO), ESA, Instituto Politécnico de Bragança, Campus de Santa Apolónia, 1172, 5301-855 Bragança, Portugal \\ b Centro de Ciências e Tecnologias Nucleares, Instituto Superior Técnico, Universidade de Lisboa, Estrada Nacional 10 (km 139,7), 2695-066 Bobadela LRS, Portugal \\ c REQUIMTE/Depto. de Ciências Químicas, Faculdade de Farmácia, Universidade do Porto, Rua Jorge Viterbo Ferreira n. ${ }^{\circ} 228,4050-313$ Porto, Portugal
}

\section{A R T I C L E I N F O}

\section{Article history:}

Received 8 October 2014

Accepted 25 November 2014

Available online 3 December 2014

\section{Keywords:}

Gamma irradiation

Food plants

Chemical/nutritional composition

Antioxidant activity

Principal component analysis

\begin{abstract}
A B S T R A C T
Aromatic plants require effective conservation technologies to expand their use. Irradiation might ensure plant decontamination, while maintaining their chemical, organoleptic, nutritional and bioactive qualities. In this study, the effects of gamma irradiation ( 1 and $10 \mathrm{kGy}$ ) in chemical, nutritional and antioxidant properties of Aloysia citrodora, Melissa officinalis, Melittis melissophyllum and Mentha piperita were evaluated. Gamma irradiation (up to $10 \mathrm{kGy}$ ) caused some statistically significant changes. However, when analyzed under an integrated approach, unirradiated and irradiated samples were grouped indiscriminately, indicating that irradiation treatment did not cause sufficient changes to define a specific chemical profile. Interestingly, each species was differentially affected by irradiation treatment. Overall, it might be considered that gamma irradiation (up to $10 \mathrm{kGy}$ ) is a feasible conservation technology for the assayed Lamiaceae and Verbenaceae species. This is an interesting result because the $10 \mathrm{kGy}$ dose guarantees disinfested and decontaminated samples.
\end{abstract}

(c) 2014 Elsevier Ltd. All rights reserved.

\section{Introduction}

Aloysia citrodora P., Melissa officinalis L., Melittis melissophyllum L. and Mentha piperita L. are widely consumed in infusions and other beverages, being also included as ingredients in many other food products (e.g., salads, sauces, marinades, ice-creams, flavoring jams and jellies, cheese, etc.) (Small, 1996). Besides aromatic and culinary purposes, their infusions are used for gastrointestinal and nervous system disorders, displaying antioxidant, antimicrobial and anti-inflammatory properties (Kapp et al., 2013; Ragone, Sella, Conforti, Volonté, \& Consolini, 2007; Skrzypczak-Pietraszeka \& Pietraszek, 2012).

Currently, the plants used in food products or dietary supplements gather special interest. Their inclusion in food formulations requires stringent regulations, starting by an irreproachable microbiological quality of raw materials (Haleem, Salem, Fatahallah, \& Abdelfattah, 2014; Ibrahim, Mohammed, Isah, \& Aliyu, 2014). This might be achieved by decontamination methods that should be safe, fast and effective against microorganisms, without changing the organoleptic and chemical characteristics of the plant (Migdal \& Owczarczyk, 1998). Hence, it is important to verify the maintenance of individual compounds such as fatty acids, tocopherols, organic acids or free sugars, besides ensuring that physical parameters are kept unchanged in the samples submitted to the decontamination treatments. Likewise, the bioactive properties of

\footnotetext{
* Corresponding author. Tel.: + 351 273303219; fax: + 351273325405.

E-mail address: iferreira@ipb.pt (I.C.F.R. Ferreira).
}

the final products should at least maintain the effectiveness of the starting materials (Nagy, Solar, Sontag, \& Koenig, 2011).

One of the decontamination techniques used for plants with food applications is irradiation. This method, besides being recommended for dry ingredients, reduces reliance on chemical fumigants (which are carcinogens and mutagens to humans, leave chemical residue on plant and destroy the ozone layer in the atmosphere) (Chmielewski \& Migdał, 2005; Migdal \& Owczarczyk, 1998). It is also characterized for its efficiency in storage, reducing losses caused by natural physiological processes (budding, maturation and aging), and eliminating or reducing microorganisms, parasites and pests without causing significant changes (chemical or organoleptic), making the plants safer for consumers (Byun, Yook, Kim, \& Chung, 1999; Nagy et al., 2011).

The aim of this work is to evaluate the effects of gamma irradiation (at 1 and $10 \mathrm{kGy}$ doses) on chemical, nutritional and antioxidant properties of A. citrodora, M. officinalis, M. melissophyllum and M. piperita.

\section{Materials and methods}

\subsection{Samples and sample irradiation}

Samples of A. citrodora P. (Verbenaceae; lemon verbena), M. officinalis L. (Lamiaceae; lemon balm), M. melissophyllum L. (Lamiaceae; bastard balm) and M. piperita L. (Lamiaceae; peppermint) were provided as dry leaves by a local producer (Pragmático Aroma Lda, Alfândega da Fé, Bragança, Portugal). After confirmation of the taxonomical identification, 
the samples were divided into three groups: control (unirradiated, 0 $\mathrm{kGy}$ ), group 1 and group 2, where $1 \mathrm{kGy}$ and $10 \mathrm{kGy}$ were, respectively, the predicted doses.

The irradiation was performed in a Co-60 experimental chamber (Precisa 22, Graviner Manufacturing Company Ltd., UK) with total activity 177 TBq (4.78 kCi), in September 2013 (Fernandes et al., 2013). The estimated doses, dose rates and dose uniformity ratios $\left(D_{\max } / D_{\min }\right)$ were, respectively: $1.20 \pm 0.07 \mathrm{kGy}, 2.57 \pm 0.15 \mathrm{kGy} \mathrm{h}^{-1}, 1.20$ for sample 1 and $8.93 \pm 0.14 \mathrm{kGy}, 1.91 \pm 0.03 \mathrm{kGy} \mathrm{h}^{-1}, 1.02$ for sample 2 . For simplicity, the values 0,1 and $10 \mathrm{kGy}$ were considered as the doses of unirradiated and irradiated groups 1 and 2, respectively.

After irradiation, the samples were grinded to powder (20 mesh) and mixed to obtain homogenized samples for subsequent analysis.

\subsection{Standards and reagents}

\subsubsection{For irradiation}

A Fricke dosimeter (chemical solution sensitive to ionizing radiation) prepared in the lab following the standards (ASTM, 1992) and Amber Perspex dosimeters (batch V, from Harwell Company, UK) were used to estimate the dose and dose rate of irradiation. To prepare the acid aqueous Fricke dosimeter solution, the following reagents were used: ferrous ammonium sulfate(II) hexahydrate, sodium chloride and sulfuric acid, all purchased from Panreac S.A. (Barcelona, Spain) with purity PA (proanalysis), and water treated in a Milli-Q water purification system (Millipore, model A10, USA).

\subsubsection{For chemical analyses}

Acetonitrile 99.9\%, n-hexane 95\% and ethyl acetate 99.8\% were of HPLC grade from Fisher Scientific (Lisbon, Portugal). Fatty acid methyl ester (FAME) reference standard mixture 37 (standard 47885-U) was purchased from Sigma (St. Louis, MO, USA), as well as other individual fatty acid isomers, L-ascorbic acid, tocopherol, sugar and organic acid standards. Racemic tocol, $50 \mathrm{mg} / \mathrm{mL}$, was purchased from Matreya (Pleasant Gap, PA, USA).

\subsection{Proximate analysis}

Protein, fat, carbohydrates and ash were determined following the AOAC procedures (AOAC, 1995). The crude protein content $(\mathrm{N} \times 6.25)$ was estimated by the macro-Kjeldahl method; the crude fat was determined using a Soxhlet apparatus by extracting (during $12 \mathrm{~h}$ ) a known weight ( $\approx 5 \mathrm{~g}$ ) of sample with petroleum ether; the ash content was determined by incineration at $600 \pm 15^{\circ} \mathrm{C}$, until a whitish ash was formed. Total carbohydrates were calculated by difference and total energy was calculated according to the following equation:

Energy $($ kcal $)=4 \times\left(\mathrm{g}_{\text {protein }}+\mathrm{g}_{\text {carbohydrates }}\right)+9 \times\left(\mathrm{g}_{\mathrm{fat}}\right)$.

\subsection{Color Measurement}

A colorimeter (model CR-400, from Konica Minolta Sensing, Inc., Japan), with an adapter for granular materials (model CR-A50) was used to measure the color of the samples. Using the illuminant $C$ and diaphragm aperture of $8 \mathrm{~mm}$, the CIE $L^{*} a^{*} b^{*}$ color space values were registered using a data software "Spectra Magic Nx" (version CM-S100W 2.03.0006), from Konica Minolta company (Japan). Before starting the measurements the instrument was calibrated against a standard white tile (Fernandes et al., 2012).

The color of three samples from each batch was measured in three different points, for each dose and at each time point, being considered the average value.

Table 1

Proximate composition and color parameters ( $L^{*}$ : lightness, $\mathrm{a}^{*}$ : redness, $\mathrm{b}^{*}$ : yellowness) of the four assayed species submitted to gamma irradiation (GI). ${ }^{1}$

\begin{tabular}{|c|c|c|c|c|c|c|c|c|c|}
\hline & & $\begin{array}{l}\text { Fat } \\
(\mathrm{g} / 100 \mathrm{~g} \mathrm{fw})\end{array}$ & $\begin{array}{l}\text { Protein } \\
(\mathrm{g} / 100 \mathrm{~g} \mathrm{dw})\end{array}$ & $\begin{array}{l}\text { Ash } \\
(\mathrm{g} / 100 \mathrm{~g} \mathrm{dw})\end{array}$ & $\begin{array}{l}\text { Carbohydrates } \\
\text { (g/100 g dw) }\end{array}$ & $\begin{array}{l}\text { Energy } \\
\text { (kcal/100 g dw) }\end{array}$ & $L^{*}$ & $a^{*}$ & $b^{*}$ \\
\hline \multicolumn{10}{|c|}{ Aloysia citrodora } \\
\hline \multirow[t]{3}{*}{ GI } & 0 kGy & $1.6 \pm 0.1^{\mathrm{b}}$ & $3.0 \pm 0.1^{\mathrm{a}}$ & $8.2 \pm 0.1^{b}$ & $87.1 \pm 0.1^{\mathrm{b}}$ & $375 \pm 1^{\mathrm{b}}$ & $49 \pm 1^{b}$ & $-8.4 \pm 0.2$ & $27.2 \pm 0.3^{b}$ \\
\hline & $1 \mathrm{kGy}$ & $2.1 \pm 0.1^{\mathrm{a}}$ & $1.8 \pm 0.1^{\mathrm{b}}$ & $8.5 \pm 0.3^{\mathrm{a}}$ & $87.6 \pm 0.4^{\mathrm{a}}$ & $377 \pm 1^{\mathrm{a}}$ & $50 \pm 1^{a}$ & $-8.8 \pm 0.3$ & $28.0 \pm 0.4^{\mathrm{a}}$ \\
\hline & 10 kGy & $1.7 \pm 0.1^{\mathrm{b}}$ & $3.0 \pm 0.2^{\mathrm{a}}$ & $8.6 \pm 0.2^{\mathrm{a}}$ & $86.7 \pm 0.1^{\mathrm{c}}$ & $374 \pm 1^{\mathrm{c}}$ & $48 \pm 1^{\mathrm{b}}$ & $-8 \pm 1$ & $26.4 \pm 0.4^{\mathrm{c}}$ \\
\hline \multirow[t]{3}{*}{$p$-values } & Homoscedasticity $^{2}$ & 0.471 & 0.323 & 0.001 & 0.003 & 0.074 & 0.495 & 0.031 & 0.951 \\
\hline & Normal distribution $^{3}$ & 0.001 & $<0.001$ & 0.016 & 0.033 & 0.125 & 0.110 & $<0.001$ & 0.612 \\
\hline & 1-way ANOVA ${ }^{4}$ & $<0.001$ & $<0.001$ & 0.004 & $<0.001$ & $<0.001$ & $<0.001$ & 0.100 & $<0.001$ \\
\hline \multicolumn{10}{|c|}{ Melissa officinalis } \\
\hline \multirow[t]{3}{*}{ GI } & 0 kGy & $1.2 \pm 0.1^{\mathrm{b}}$ & $2.5 \pm 0.3^{\mathrm{b}}$ & $8.4 \pm 0.4$ & $88 \pm 1^{\mathrm{a}}$ & $372 \pm 2^{c}$ & $48 \pm 1$ & $-5.1 \pm 0.5$ & $20.9 \pm 0.4^{\mathrm{a}}$ \\
\hline & $1 \mathrm{kGy}$ & $1.9 \pm 0.1^{\mathrm{a}}$ & $7 \pm 1^{a}$ & $8.1 \pm 0.3$ & $83 \pm 1^{b}$ & $377 \pm 1^{\mathrm{a}}$ & $48 \pm 1$ & $-5.1 \pm 0.5$ & $20.9 \pm 0.4^{\mathrm{a}}$ \\
\hline & $10 \mathrm{kGy}$ & $1.8 \pm 0.1^{\mathrm{a}}$ & $6 \pm 1^{\mathrm{a}}$ & $8.4 \pm 0.2$ & $83 \pm 1^{b}$ & $376 \pm 1^{\mathrm{b}}$ & $47 \pm 1$ & $-5.0 \pm 0.5$ & $20.3 \pm 0.5^{\mathrm{b}}$ \\
\hline \multirow[t]{3}{*}{$p$-values } & Homoscedasticity $^{2}$ & 0.113 & 0.003 & 0.054 & 0.002 & 0.004 & 0.191 & 0.926 & 0.412 \\
\hline & Normal distribution $^{3}$ & $<0.001$ & 0.005 & 0.145 & 0.002 & 0.037 & 0.346 & 0.703 & 0.096 \\
\hline & 1-way ANOVA 4 & $<0.001$ & $<0.001$ & 0.072 & $<0.001$ & $<0.001$ & 0.269 & 0.926 & 0.022 \\
\hline \multicolumn{10}{|c|}{ Melittis melissophyllum } \\
\hline \multirow[t]{3}{*}{ GI } & $0 \mathrm{kGy}$ & $1.8 \pm 0.1^{\mathrm{a}}$ & $4.6 \pm 0.2^{\mathrm{b}}$ & $7.6 \pm 0.1^{\mathrm{c}}$ & $86.0 \pm 0.4^{\mathrm{b}}$ & $378 \pm 1^{\mathrm{a}}$ & $42 \pm 2$ & $-8.4 \pm 0.5$ & $18 \pm 3$ \\
\hline & 1 kGy & $1.6 \pm 0.1^{\mathrm{b}}$ & $2.6 \pm 0.1^{\mathrm{c}}$ & $8.1 \pm 0.1^{\mathrm{b}}$ & $87.7 \pm 0.2^{\mathrm{a}}$ & $376 \pm 1^{\mathrm{b}}$ & $44 \pm 2$ & $-8.2 \pm 0.5$ & $17 \pm 1$ \\
\hline & $10 \mathrm{kGy}$ & $1.5 \pm 0.1^{\mathrm{b}}$ & $5.6 \pm 0.5^{\mathrm{a}}$ & $8.6 \pm 0.2^{\mathrm{a}}$ & $84 \pm 1^{\mathrm{c}}$ & $373 \pm 1^{\mathrm{c}}$ & $41 \pm 2$ & $-8.0 \pm 0.5$ & $16 \pm 1$ \\
\hline \multirow[t]{3}{*}{$p$-values } & Homoscedasticity $^{2}$ & 0.007 & $<0.001$ & 0.108 & $<0.001$ & 0.002 & 0.811 & 0.555 & 0.053 \\
\hline & Normal distribution $^{3}$ & 0.056 & 0.004 & 0.124 & 0.057 & 0.291 & 0.090 & 0.588 & $<0.001$ \\
\hline & 1-way ANOVA ${ }^{4}$ & $<0.001$ & $<0.001$ & $<0.001$ & $<0.001$ & $<0.001$ & 0.055 & 0.311 & 0.381 \\
\hline \multicolumn{10}{|c|}{ Mentha piperita } \\
\hline \multirow[t]{3}{*}{ GI } & 0 kGy & $2.4 \pm 0.1^{\mathrm{b}}$ & $5.1 \pm 0.3^{\mathrm{b}}$ & $9.2 \pm 0.2^{\mathrm{a}}$ & $83.3 \pm 0.5^{\mathrm{b}}$ & $375 \pm 1^{\mathrm{b}}$ & $40 \pm 1^{\mathrm{a}}$ & $-5.9 \pm 0.1^{\mathrm{a}}$ & $23.9 \pm 0.3^{\mathrm{a}}$ \\
\hline & 1 kGy & $2.7 \pm 0.2^{\mathrm{a}}$ & $3.1 \pm 0.1^{\mathrm{c}}$ & $8.4 \pm 0.1^{c}$ & $85.8 \pm 0.3^{\mathrm{a}}$ & $380 \pm 1^{\mathrm{a}}$ & $39 \pm 1^{\mathrm{a}}$ & $-5.7 \pm 0.2^{\mathrm{a}}$ & $23.2 \pm 0.5^{\mathrm{a}}$ \\
\hline & $10 \mathrm{kGy}$ & $2.0 \pm 0.2^{c}$ & $10.5 \pm 0.3^{a}$ & $8.6 \pm 0.1^{b}$ & $78.9 \pm 0.4^{\mathrm{c}}$ & $375 \pm 1^{\mathrm{b}}$ & $37 \pm 1^{\mathrm{b}}$ & $-4.8 \pm 0.4^{\mathrm{b}}$ & $20.7 \pm 0.5^{b}$ \\
\hline \multirow[t]{3}{*}{$p$-values } & Homoscedasticity $^{2}$ & 0.169 & $<0.001$ & $<0.001$ & 0.379 & 0.006 & 0.515 & 0.072 & 0.036 \\
\hline & Normal distribution $^{3}$ & 0.448 & $<0.001$ & 0.010 & 0.001 & $<0.001$ & 0.406 & 0.008 & 0.005 \\
\hline & 1-way ANOVA ${ }^{4}$ & $<0.001$ & $<0.001$ & $<0.001$ & $<0.001$ & $<0.001$ & $<0.001$ & $<0.001$ & $<0.001$ \\
\hline
\end{tabular}

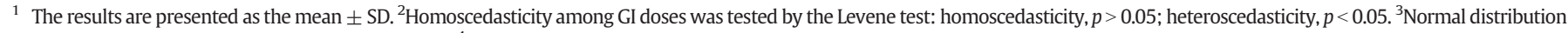

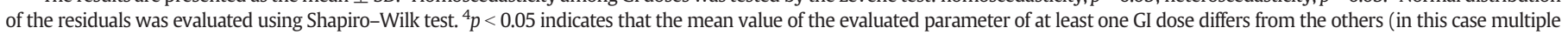
comparison tests were performed). For each species, means within a column with different letters differ significantly $(p<0.05)$. 
Table 2

Hydrophilic compounds (free sugars and organic acids) composition ( $\mathrm{g} / 100 \mathrm{~g} \mathrm{dw}$ ) of the four assayed species submitted to gamma irradiation (GI). The results are presented as mean $\pm \mathrm{SD}^{1}$.

\begin{tabular}{|c|c|c|c|c|c|c|c|c|c|c|c|c|c|}
\hline & & Fructose & Glucose & Sucrose & Trehalose & Unknown & Total sugars & Oxalic acid & Quinic acid & Malic acid & Shikimic acid & Citric acid & Total organic acids \\
\hline \multicolumn{14}{|c|}{ Aloysia citrodora } \\
\hline \multirow[t]{3}{*}{ GI } & $0 \mathrm{kGy}$ & $1.0 \pm 0.1$ & $1.3 \pm 0.1$ & $7.1 \pm 0.3^{\mathrm{a}}$ & $1.2 \pm 0.1$ & nd & $10.7 \pm 0.4^{\mathrm{a}}$ & $1.1 \pm 0.1$ & nd & $0.14 \pm 0.03^{\mathrm{b}}$ & $1.4 \pm 0.1^{\mathrm{c}}$ & $1.4 \pm 0.1^{\mathrm{c}}$ & $4.1 \pm 0.1^{\mathrm{c}}$ \\
\hline & $1 \mathrm{kGy}$ & $1.0 \pm 0.1$ & $1.2 \pm 0.1$ & $6.4 \pm 0.3^{\mathrm{b}}$ & $1.2 \pm 0.1$ & nd & $9.8 \pm 0.4^{\mathrm{b}}$ & $1.1 \pm 0.1$ & nd & $0.17 \pm 0.02^{\mathrm{a}}$ & $1.8 \pm 0.1^{\mathrm{a}}$ & $2.0 \pm 0.2^{\mathrm{a}}$ & $5.1 \pm 0.3^{\mathrm{a}}$ \\
\hline & $10 \mathrm{kGy}$ & $1.0 \pm 0.1$ & $1.2 \pm 0.1$ & $6.6 \pm 0.3^{\mathrm{b}}$ & $1.2 \pm 0.1$ & nd & $10.0 \pm 0.5^{\mathrm{b}}$ & $1.1 \pm 0.1$ & nd & $0.13 \pm 0.02^{\mathrm{b}}$ & $1.6 \pm 0.1^{\mathrm{b}}$ & $1.7 \pm 0.1^{\mathrm{b}}$ & $4.6 \pm 0.3^{\mathrm{b}}$ \\
\hline \multirow[t]{3}{*}{$p$-values } & Homoscedasticity $^{2}$ & 0.115 & 0.072 & 0.818 & 0.011 & - & 0.944 & 0.401 & - & 0.190 & 0.625 & 0.034 & 0.154 \\
\hline & Normal distribution ${ }^{3}$ & 0.672 & 0.333 & 0.308 & 0.319 & - & 0.799 & 0.288 & - & 0.481 & 0.281 & 0.184 & 0.140 \\
\hline & 1-way ANOVA ${ }^{4}$ & 0.882 & 0.065 & $<0.001$ & 0.843 & - & 0.001 & 0.233 & - & 0.007 & $<0.001$ & $<0.001$ & $<0.001$ \\
\hline \multicolumn{14}{|c|}{ Melissa officinalis } \\
\hline \multirow[t]{3}{*}{ GI } & $0 \mathrm{kGy}$ & $1.2 \pm 0.1^{\mathrm{b}}$ & $1.0 \pm 0.1$ & $4.8 \pm 0.2^{c}$ & $0.49 \pm 0.05^{c}$ & nd & $7.5 \pm 0.2^{\mathrm{c}}$ & $0.5 \pm 0.1$ & $0.26 \pm 0.04$ & $0.4 \pm 0.1$ & $4.1 \pm 0.2$ & nd & $5.3 \pm 0.3$ \\
\hline & $1 \mathrm{kGy}$ & $1.4 \pm 0.1^{\mathrm{a}}$ & $1.0 \pm 0.1$ & $5.4 \pm 0.2^{\mathrm{b}}$ & $0.67 \pm 0.03^{\mathrm{b}}$ & nd & $8.4 \pm 0.3^{b}$ & $0.5 \pm 0.1$ & $0.23 \pm 0.03$ & $0.4 \pm 0.1$ & $4.1 \pm 0.4$ & nd & $5.3 \pm 0.4$ \\
\hline & $10 \mathrm{kGy}$ & $1.3 \pm 0.1^{\mathrm{ab}}$ & $1.0 \pm 0.1$ & $5.6 \pm 0.2^{\mathrm{a}}$ & $0.85 \pm 0.05^{\mathrm{a}}$ & nd & $8.8 \pm 0.4^{\mathrm{a}}$ & $0.5 \pm 0.1$ & $0.24 \pm 0.04$ & $0.4 \pm 0.1$ & $4.1 \pm 0.4$ & nd & $5.3 \pm 0.4$ \\
\hline \multirow[t]{3}{*}{$p$-values } & Homoscedasticity $^{2}$ & 0.045 & 0.051 & 0.931 & 0.009 & - & 0.680 & 0.836 & 0.745 & 0.393 & 0.059 & - & 0.540 \\
\hline & Normal distribution ${ }^{3}$ & 0.357 & 0.167 & 0.361 & 0.440 & - & 0.684 & 0.179 & 0.140 & 0.121 & 0.115 & - & 0.073 \\
\hline & 1-way ANOVA ${ }^{4}$ & 0.004 & 0.832 & $<0.001$ & $<0.001$ & - & $<0.001$ & 0.818 & 0.185 & 0.540 & 0.986 & - & 0.929 \\
\hline \multicolumn{14}{|c|}{ Melittis melissophyllum } \\
\hline \multirow[t]{3}{*}{ GI } & $0 \mathrm{kGy}$ & $1.0 \pm 0.1$ & $0.8 \pm 0.1$ & $0.9 \pm 0.1$ & $0.28 \pm 0.03^{\mathrm{c}}$ & $2.5 \pm 0.1^{\mathrm{b}}$ & $5.5 \pm 0.3^{b}$ & $1.4 \pm 0.1^{\mathrm{a}}$ & $0.17 \pm 0.01^{\mathrm{ab}}$ & $6.0 \pm 0.3^{\mathrm{a}}$ & $0.97 \pm 0.05^{\mathrm{a}}$ & $0.022 \pm 0.001^{\mathrm{b}}$ & $8.6 \pm 0.4^{\mathrm{a}}$ \\
\hline & $1 \mathrm{kGy}$ & $0.9 \pm 0.1$ & $0.8 \pm 0.1$ & $0.9 \pm 0.1$ & $0.53 \pm 0.05^{\mathrm{b}}$ & $2.7 \pm 0.1^{\mathrm{a}}$ & $5.9 \pm 0.4^{\mathrm{b}}$ & $1.2 \pm 0.1^{\mathrm{b}}$ & $0.15 \pm 0.02^{\mathrm{b}}$ & $4.5 \pm 0.2^{\mathrm{b}}$ & $0.86 \pm 0.05^{\mathrm{b}}$ & $0.019 \pm 0.001^{\mathrm{c}}$ & $6.6 \pm 0.3^{\mathrm{b}}$ \\
\hline & $10 \mathrm{kGy}$ & $1.0 \pm 0.1$ & $0.9 \pm 0.1$ & $1.0 \pm 0.1$ & $0.63 \pm 0.05^{\mathrm{a}}$ & $2.8 \pm 0.1^{\mathrm{a}}$ & $6.3 \pm 0.3^{\mathrm{a}}$ & $1.4 \pm 0.1^{\mathrm{a}}$ & $0.19 \pm 0.01^{\mathrm{a}}$ & $5.9 \pm 0.3^{\mathrm{a}}$ & $0.95 \pm 0.05^{\mathrm{a}}$ & $0.026 \pm 0.002^{\mathrm{a}}$ & $8.5 \pm 0.4^{\mathrm{a}}$ \\
\hline \multirow[t]{3}{*}{$p$-values } & Homoscedasticity $^{2}$ & 0.495 & 0.954 & 0.040 & $<0.001$ & 0.709 & 0.431 & 0.921 & 0.630 & 0.269 & 0.902 & 0.058 & 0.378 \\
\hline & Normal distribution ${ }^{3}$ & 0.270 & 0.759 & 0.005 & 0.012 & 0.799 & 0.681 & 0.054 & 0.839 & 0.002 & 0.998 & 0.113 & 0.005 \\
\hline & 1-way ANOVA ${ }^{4}$ & 0.052 & 0.055 & 0.072 & $<0.001$ & 0.001 & $<0.001$ & $<0.001$ & $<0.001$ & $<0.001$ & 0.002 & $<0.001$ & $<0.001$ \\
\hline \multicolumn{14}{|c|}{ Mentha piperita } \\
\hline \multirow{3}{*}{ GI } & $0 \mathrm{kGy}$ & $0.47 \pm 0.05^{\mathrm{a}}$ & $0.30 \pm 0.05$ & $0.7 \pm 0.1$ & $1.0 \pm 0.1^{\mathrm{a}}$ & nd & $2.4 \pm 0.2$ & $1.1 \pm 0.1^{\mathrm{a}}$ & $0.040 \pm 0.003^{\mathrm{a}}$ & $0.9 \pm 0.1^{\mathrm{a}}$ & nd & $8.5 \pm 0.2^{\mathrm{a}}$ & $10.6 \pm 0.3^{\mathrm{a}}$ \\
\hline & $1 \mathrm{kGy}$ & $0.42 \pm 0.03^{b}$ & $0.29 \pm 0.03$ & $0.8 \pm 0.1$ & $1.0 \pm 0.1^{\mathrm{a}}$ & nd & $2.5 \pm 0.2$ & $1.2 \pm 0.1^{\mathrm{a}}$ & $0.036 \pm 0.004^{\mathrm{ab}}$ & $0.9 \pm 0.1^{\mathrm{a}}$ & nd & $6.5 \pm 0.2^{\mathrm{c}}$ & $8.7 \pm 0.2^{\mathrm{c}}$ \\
\hline & $10 \mathrm{kGy}$ & $0.47 \pm 0.04^{\mathrm{ab}}$ & $0.31 \pm 0.03$ & $0.7 \pm 0.1$ & $0.8 \pm 0.1^{\mathrm{b}}$ & nd & $2.3 \pm 0.2$ & $1.0 \pm 0.1^{\mathrm{b}}$ & $0.035 \pm 0.003^{\mathrm{b}}$ & $0.7 \pm 0.1^{\mathrm{b}}$ & nd & $7.7 \pm 0.2^{\mathrm{b}}$ & $9.5 \pm 0.2^{\mathrm{b}}$ \\
\hline \multirow[t]{3}{*}{$p$-values } & Homoscedasticity $^{2}$ & 0.665 & 0.061 & 0.131 & 0.320 & - & 0.573 & 0.934 & 0.880 & 0.880 & - & 0.559 & 0.039 \\
\hline & Normal distribution ${ }^{3}$ & 0.767 & 0.240 & 0.818 & 0.626 & - & 0.681 & 0.178 & 0.196 & 0.016 & - & 0.046 & $<0.001$ \\
\hline & 1 -way ANOVA ${ }^{4}$ & 0.030 & 0.507 & 0.060 & $<0.001$ & - & 0.094 & $<0.001$ & 0.013 & $<0.001$ & - & $<0.001$ & $<0.001$ \\
\hline
\end{tabular}

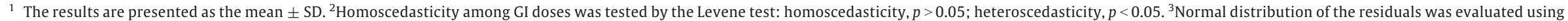

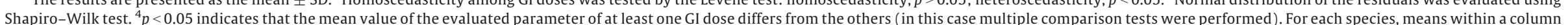
with different letters differ significantly $(p<0.05)$. 


\subsection{Chemical composition of hydrophilic compounds}

\subsubsection{Sugars}

Free sugars were determined by high performance liquid chromatography coupled to a refraction index detector (HPLC-RI). Dried sample powder $(1.0 \mathrm{~g})$ was spiked with melezitose as internal standard (IS, $5 \mathrm{mg} / \mathrm{mL}$ ), and extracted with $40 \mathrm{~mL}$ of $80 \%$ aqueous ethanol at $80{ }^{\circ} \mathrm{C}$ for $30 \mathrm{~min}$. The resulting suspension was centrifuged (Centurion K24OR refrigerated centrifuge, West Sussex, UK) at 15,000 $\mathrm{g}$ for $10 \mathrm{~min}$. The supernatant was concentrated at $60^{\circ} \mathrm{C}$ under reduced pressure and defatted three times with $10 \mathrm{~mL}$ of ethyl ether, successively. After concentration at $40{ }^{\circ} \mathrm{C}$, the solid residues were dissolved in water to a final volume of $5 \mathrm{~mL}$ and filtered through $0.2 \mu \mathrm{m}$ Whatman nylon filters. Chromatographic conditions were applied as previously defined (Barros et al., 2013). The compounds were identified by chromatographic comparisons with authentic standards. Quantification was performed using the internal standard method and sugar contents were further expressed in $\mathrm{g} / 100 \mathrm{~g}$ of dry weight (dw).

\subsubsection{Organic acids}

Organic acids were determined following a procedure previously described by the authors. Samples $(\approx 2 \mathrm{~g})$ were extracted by stirring with $25 \mathrm{~mL}$ of meta-phosphoric acid $\left(25{ }^{\circ} \mathrm{C}\right.$ at $150 \mathrm{rpm}$ ) for $45 \mathrm{~min}$ and subsequently filtered through Whatman No. 4 paper. Before analysis, the sample was filtered through $0.2 \mu$ m nylon filters. Chromatographic conditions were applied as previously defined (Barros et al., 2013). Detection was carried out in a DAD, using $215 \mathrm{~nm}$ and $245 \mathrm{~nm}$ (for ascorbic acid) as preferred wavelengths. The organic acids found were quantified by comparison of the area of their peaks recorded at $215 \mathrm{~nm}$ with calibration curves obtained from commercial standards of each compound.

\subsection{Chemical composition in lipophilic compounds}

\subsubsection{Tocopherols}

Tocopherols were determined following a procedure previously described by the authors (Pereira, Barros, \& Ferreira, 2013). The compounds were identified by chromatographic comparisons with authentic standards. Quantification was based on the fluorescence signal response of each standard, using the IS (tocol) method and by using calibration curves obtained from commercial standards of each compound.

\subsubsection{Fatty acids}

Fatty acids were determined by gas-liquid chromatography with flame ionization detection (GC-FID)/capillary column as described previously by the authors (Pereira et al., 2013). Fatty acid identification was made by comparing the relative retention times of FAME peaks from samples with standards. The results were recorded and processed using the CSW 1.7 Software (DataApex 1.7, Prague, Czech Republic).

\subsection{Evaluation of bioactivity}

\subsubsection{Samples preparation}

The methanolic extracts were obtained from the dried plant material. The sample $(1 \mathrm{~g})$ was extracted by stirring with $25 \mathrm{~mL}$ of methanol $\left(25^{\circ} \mathrm{C}\right.$ at $150 \mathrm{rpm}$ ) for $1 \mathrm{~h}$ and subsequently filtered through Whatman No. 4 paper. The residue was then extracted with $25 \mathrm{~mL}$ of methanol $\left(25^{\circ} \mathrm{C}\right.$ at $150 \mathrm{rpm}$ ) for $1 \mathrm{~h}$. The combined methanolic extracts were evaporated at $40{ }^{\circ} \mathrm{C}$ (rotary evaporator Büchi R-210, Flawil, Switzerland) to dryness.

The infusions were also obtained from the dried plant material. The sample ( $1 \mathrm{~g}$ ) was added to $200 \mathrm{~mL}$ of boiling distilled water (after being taken out from the heating source) and left to stand at room temperature for $5 \mathrm{~min}$, and then filtered under reduced pressure. The obtained infusions were frozen and lyophilized.

Table 3

Tocopherols composition ( $\mathrm{mg} / 100 \mathrm{~g} \mathrm{dw}$ ) of the four assayed species submitted to gamma irradiation (GI). The results are presented as mean $\pm \mathrm{SD}^{1}$.

\begin{tabular}{|c|c|c|c|c|c|c|}
\hline & & $\alpha$-Tocopherol & $\beta$-Tocopherol & $\gamma$-Tocopherol & $\delta$-Tocopherol & Total tocopherols \\
\hline \multicolumn{7}{|c|}{ Aloysia citrodora } \\
\hline \multirow[t]{3}{*}{ GI } & $0 \mathrm{kGy}$ & $15.3 \pm 0.4^{\mathrm{b}}$ & $0.41 \pm 0.04^{\mathrm{a}}$ & $1.8 \pm 0.1^{\mathrm{ab}}$ & nd & $17.5 \pm 0.4^{\mathrm{b}}$ \\
\hline & 1 kGy & $17.5 \pm 0.4^{\mathrm{a}}$ & $0.44 \pm 0.05^{\mathrm{a}}$ & $1.9 \pm 0.1^{\mathrm{a}}$ & nd & $19.8 \pm 0.4^{\mathrm{a}}$ \\
\hline & $10 \mathrm{kGy}$ & $13.4 \pm 0.3^{\mathrm{c}}$ & $0.29 \pm 0.04^{b}$ & $1.7 \pm 0.1^{\mathrm{b}}$ & nd & $15.4 \pm 0.3^{c}$ \\
\hline \multirow[t]{3}{*}{$p$-values } & Homoscedasticity $^{2}$ & 0.831 & 0.012 & 0.341 & - & 0.412 \\
\hline & Normal distribution $^{3}$ & 0.024 & 0.378 & 0.352 & - & 0.020 \\
\hline & 1-way ANOVA ${ }^{4}$ & $<0.001$ & $<0.001$ & 0.002 & - & $<0.001$ \\
\hline \multicolumn{7}{|c|}{ Melissa officinalis } \\
\hline \multirow[t]{3}{*}{ GI } & 0 kGy & $29 \pm 1^{b}$ & $1.3 \pm 0.1^{\mathrm{a}}$ & $1.5 \pm 0.1^{\mathrm{b}}$ & $0.37 \pm 0.05^{\mathrm{b}}$ & $32 \pm 1^{\mathrm{b}}$ \\
\hline & $1 \mathrm{kGy}$ & $33 \pm 1^{\mathrm{a}}$ & $1.1 \pm 0.1^{\mathrm{b}}$ & $1.8 \pm 0.1^{\mathrm{a}}$ & $0.38 \pm 0.05^{\mathrm{b}}$ & $37 \pm 1^{\mathrm{a}}$ \\
\hline & 10 kGy & $29 \pm 1^{b}$ & $0.9 \pm 0.1^{\mathrm{c}}$ & $1.7 \pm 0.1^{\mathrm{a}}$ & $0.49 \pm 0.05^{\mathrm{a}}$ & $33 \pm 1^{b}$ \\
\hline \multirow[t]{3}{*}{$p$-values } & Homoscedasticity $^{2}$ & 0.646 & 0.017 & 0.264 & 0.215 & 0.671 \\
\hline & Normal distribution $^{3}$ & 0.001 & 0.139 & 0.553 & 0.151 & 0.003 \\
\hline & 1-way ANOVA ${ }^{4}$ & $<0.001$ & $<0.001$ & $<0.001$ & 0.001 & $<0.001$ \\
\hline \multicolumn{7}{|c|}{ Melittis melissophyllum } \\
\hline \multirow[t]{3}{*}{ GI } & $0 \mathrm{kGy}$ & $0.88 \pm 0.05^{\mathrm{a}}$ & $13.4 \pm 0.3^{\mathrm{b}}$ & $0.18 \pm 0.02^{\mathrm{a}}$ & $0.14 \pm 0.02^{\mathrm{a}}$ & $14.6 \pm 0.4^{\mathrm{b}}$ \\
\hline & 1 kGy & $0.81 \pm 0.05^{\mathrm{b}}$ & $13.2 \pm 0.2^{\mathrm{b}}$ & $0.16 \pm 0.02^{\mathrm{a}}$ & $0.14 \pm 0.02^{\mathrm{a}}$ & $14.3 \pm 0.2^{\mathrm{b}}$ \\
\hline & $10 \mathrm{kGy}$ & $0.46 \pm 0.04^{\mathrm{c}}$ & $28.9 \pm 0.3^{\mathrm{a}}$ & $0.11 \pm 0.02^{\mathrm{b}}$ & $0.08 \pm 0.01^{\mathrm{b}}$ & $29.5 \pm 0.2^{\mathrm{a}}$ \\
\hline \multirow[t]{3}{*}{$p$-values } & Homoscedasticity $^{2}$ & 0.073 & 0.501 & 0.423 & 0.245 & 0.481 \\
\hline & Normal distribution $^{3}$ & 0.001 & $<0.001$ & 0.386 & 0.180 & $<0.001$ \\
\hline & 1-way ANOVA ${ }^{4}$ & $<0.001$ & $<0.001$ & $<0.001$ & $<0.001$ & $<0.001$ \\
\hline \multicolumn{7}{|c|}{ Mentha piperita } \\
\hline \multirow[t]{3}{*}{ GI } & 0 kGy & $16.5 \pm 0.4^{\mathrm{a}}$ & $1.1 \pm 0.1^{\mathrm{a}}$ & $1.8 \pm 0.1$ & $0.23 \pm 0.03^{\mathrm{b}}$ & $19.7 \pm 0.5^{\mathrm{a}}$ \\
\hline & $1 \mathrm{kGy}$ & $15.7 \pm 0.2^{\mathrm{b}}$ & $0.8 \pm 0.1^{\mathrm{b}}$ & $1.8 \pm 0.1$ & $0.28 \pm 0.04^{\mathrm{a}}$ & $18.6 \pm 0.2^{\mathrm{b}}$ \\
\hline & 10 kGy & $13.2 \pm 0.2^{\mathrm{c}}$ & $0.9 \pm 0.1^{\mathrm{b}}$ & $1.8 \pm 0.1$ & $0.30 \pm 0.03^{\mathrm{a}}$ & $16.2 \pm 0.4^{\mathrm{c}}$ \\
\hline \multirow[t]{3}{*}{$p$-values } & Homoscedasticity $^{2}$ & 0.002 & 0.064 & 0.778 & 0.427 & 0.001 \\
\hline & Normal distribution $^{3}$ & 0.001 & 0.012 & 0.187 & 0.559 & 0.021 \\
\hline & 1-way ANOVA ${ }^{4}$ & $<0.001$ & $<0.001$ & 0.797 & 0.001 & $<0.001$ \\
\hline
\end{tabular}

\footnotetext{
1 The results are presented as the mean \pm SD. ${ }^{2}$ Homoscedasticity among GI doses was tested by the Levene test: homoscedasticity, $p>0.05$; heteroscedasticity, $p<0.05 .{ }^{3}$ Normal distribution

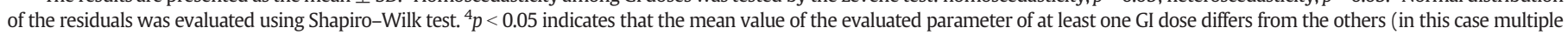
comparison tests were performed). For each species, means within a column with different letters differ significantly $(p<0.05)$.
} 
Table 4A

A. Minor fatty acids (values $<1 \%$ in all species) of the four assayed species submitted to gamma irradiation (GI). The results are presented in relative percentage as mean $\pm \mathrm{SD}^{1}$.

\begin{tabular}{|c|c|c|c|c|c|c|c|c|c|c|c|c|c|}
\hline & & C6:0 & C8:0 & C11:0 & C12:0 & C13:0 & C15:0 & $\mathrm{C} 15: 1$ & C17:0 & $\mathrm{C} 20: 1 \mathrm{n} 9$ & $\mathrm{C} 20: 2 \mathrm{n} 6$ & $\mathrm{C} 20: 3 \mathrm{n} 3+\mathrm{C} 21: 0$ & $\mathrm{C} 22: 1 \mathrm{n} 9$ \\
\hline \multicolumn{14}{|c|}{ Aloysia citrodora } \\
\hline \multirow[t]{3}{*}{ GI } & $0 \mathrm{kGy}$ & $0.30 \pm 0.01^{\mathrm{a}}$ & $0.11 \pm 0.01^{\mathrm{b}}$ & $0.26 \pm 0.02^{\mathrm{a}}$ & $0.26 \pm 0.02^{\mathrm{b}}$ & $0.32 \pm 0.01^{\mathrm{c}}$ & $0.58 \pm 0.02^{\mathrm{b}}$ & $0.10 \pm 0.01^{\mathrm{a}}$ & $0.22 \pm 0.01^{\mathrm{c}}$ & $0.25 \pm 0.03^{\mathrm{b}}$ & $0.21 \pm 0.01^{\mathrm{b}}$ & $0.30 \pm 0.01^{\mathrm{a}}$ & $0.27 \pm 0.02^{\mathrm{b}}$ \\
\hline & $1 \mathrm{kGy}$ & $0.28 \pm 0.04^{\mathrm{a}}$ & $0.10 \pm 0.01^{\mathrm{b}}$ & $0.21 \pm 0.01^{\mathrm{b}}$ & $0.29 \pm 0.02^{b}$ & $0.46 \pm 0.03^{\mathrm{a}}$ & $0.61 \pm 0.05^{\mathrm{b}}$ & $0.09 \pm 0.01^{\mathrm{b}}$ & $0.24 \pm 0.01^{\mathrm{b}}$ & $0.39 \pm 0.04^{\mathrm{a}}$ & $0.17 \pm 0.01^{\mathrm{c}}$ & $0.27 \pm 0.01^{\mathrm{c}}$ & $0.37 \pm 0.01^{\circ}$ \\
\hline & $10 \mathrm{kGy}$ & $0.23 \pm 0.02^{\mathrm{b}}$ & $0.13 \pm 0.01^{\mathrm{a}}$ & $0.24 \pm 0.03^{\mathrm{a}}$ & $0.37 \pm 0.03^{\mathrm{a}}$ & $0.35 \pm 0.02^{\mathrm{b}}$ & $0.71 \pm 0.02^{\mathrm{a}}$ & $0.10 \pm 0.01^{\mathrm{a}}$ & $0.27 \pm 0.01^{\mathrm{a}}$ & $0.22 \pm 0.02^{\mathrm{b}}$ & $0.27 \pm 0.01^{\mathrm{a}}$ & $0.28 \pm 0.01^{\mathrm{b}}$ & $0.19 \pm 0.01^{c}$ \\
\hline \multirow[t]{3}{*}{$p$-values } & Homoscedasticity $^{2}$ & $<0.001$ & 0.008 & 0.008 & 0.100 & 0.004 & 0.003 & 0.002 & 0.038 & 0.001 & 0.008 & $<0.001$ & $<0.001$ \\
\hline & Normal distribution ${ }^{3}$ & 0.015 & 0.163 & 0.210 & 0.071 & 0.003 & 0.010 & 0.038 & 0.002 & 0.001 & 0.001 & 0.001 & 0.001 \\
\hline & 1 -way ANOVA ${ }^{4}$ & $<0.001$ & 0.001 & $<0.001$ & $<0.001$ & $<0.001$ & $<0.001$ & $<0.001$ & $<0.001$ & $<0.001$ & $<0.001$ & $<0.001$ & $<0.001$ \\
\hline \multicolumn{14}{|c|}{ Melissa officinalis } \\
\hline \multirow[t]{3}{*}{ GI } & 0 kGy & $0.22 \pm 0.01^{\mathrm{a}}$ & $0.40 \pm 0.02^{\mathrm{a}}$ & $0.13 \pm 0.01^{\mathrm{b}}$ & $0.46 \pm 0.01^{\mathrm{a}}$ & $0.14 \pm 0.01^{\mathrm{b}}$ & $0.44 \pm 0.03^{\mathrm{a}}$ & $0.55 \pm 0.01^{\mathrm{a}}$ & $0.81 \pm 0.01^{\mathrm{b}}$ & $0.18 \pm 0.02^{\mathrm{a}}$ & nd & $0.28 \pm 0.01^{\mathrm{c}}$ & nd \\
\hline & $1 \mathrm{kGy}$ & $0.15 \pm 0.01^{\mathrm{b}}$ & $0.30 \pm 0.02^{\mathrm{b}}$ & $0.13 \pm 0.01^{\mathrm{b}}$ & $0.34 \pm 0.01^{\mathrm{b}}$ & $0.16 \pm 0.01^{\mathrm{a}}$ & $0.42 \pm 0.01^{\mathrm{a}}$ & $0.49 \pm 0.01^{\mathrm{c}}$ & $0.87 \pm 0.01^{\mathrm{a}}$ & $0.15 \pm 0.01^{\mathrm{b}}$ & nd & $0.35 \pm 0.01^{\mathrm{b}}$ & nd \\
\hline & $10 \mathrm{kGy}$ & $0.14 \pm 0.01^{\mathrm{c}}$ & $0.29 \pm 0.01^{\mathrm{b}}$ & $0.17 \pm 0.01^{\mathrm{a}}$ & $0.30 \pm 0.01^{\mathrm{c}}$ & $0.14 \pm 0.01^{\mathrm{b}}$ & $0.36 \pm 0.01^{\mathrm{b}}$ & $0.51 \pm 0.01^{\mathrm{b}}$ & $0.80 \pm 0.01^{\mathrm{c}}$ & $0.12 \pm 0.03^{\mathrm{b}}$ & nd & $0.36 \pm 0.01^{\mathrm{a}}$ & nd \\
\hline \multirow[t]{3}{*}{$p$-values } & Homoscedasticity $^{2}$ & 0.002 & 0.672 & 0.089 & 0.002 & $<0.001$ & $<0.001$ & $<0.001$ & 0.007 & 0.039 & - & $<0.001$ & - \\
\hline & Normal distribution ${ }^{3}$ & 0.001 & 0.001 & $<0.001$ & $<0.001$ & 0.058 & 0.006 & 0.001 & $<0.001$ & 0.500 & - & $<0.001$ & - \\
\hline & 1-way ANOVA ${ }^{4}$ & $<0.001$ & $<0.001$ & $<0.001$ & $<0.001$ & $<0.001$ & $<0.001$ & $<0.001$ & $<0.001$ & $<0.001$ & - & $<0.001$ & - \\
\hline \multicolumn{14}{|c|}{ Melittis melissophyllum } \\
\hline \multirow[t]{3}{*}{ GI } & $0 \mathrm{kGy}$ & $0.18 \pm 0.01^{\mathrm{a}}$ & $0.07 \pm 0.01^{\mathrm{b}}$ & $0.04 \pm 0.01^{\mathrm{b}}$ & $0.18 \pm 0.01^{\mathrm{b}}$ & $0.05 \pm 0.01^{\mathrm{c}}$ & $0.90 \pm 0.02^{\mathrm{b}}$ & $0.09 \pm 0.01^{\mathrm{b}}$ & $0.24 \pm 0.02$ & $0.16 \pm 0.01^{\mathrm{c}}$ & $0.09 \pm 0.02^{\mathrm{c}}$ & $0.24 \pm 0.01^{\mathrm{b}}$ & nd \\
\hline & $1 \mathrm{kGy}$ & $0.06 \pm 0.01^{\mathrm{c}}$ & $0.07 \pm 0.01^{\mathrm{b}}$ & $0.04 \pm 0.01^{\mathrm{b}}$ & $0.24 \pm 0.02^{\mathrm{a}}$ & $0.06 \pm 0.01^{\mathrm{b}}$ & $0.83 \pm 0.03^{c}$ & $0.08 \pm 0.01^{\mathrm{c}}$ & $0.24 \pm 0.01$ & $0.20 \pm 0.01^{\mathrm{a}}$ & $0.15 \pm 0.01^{\mathrm{b}}$ & $0.27 \pm 0.01^{\mathrm{a}}$ & nd \\
\hline & $10 \mathrm{kGy}$ & $0.08 \pm 0.01^{\mathrm{b}}$ & $0.09 \pm 0.01^{\mathrm{a}}$ & $0.08 \pm 0.01^{\mathrm{a}}$ & $0.25 \pm 0.01^{\mathrm{a}}$ & $0.07 \pm 0.01^{\mathrm{a}}$ & $0.96 \pm 0.02^{\mathrm{a}}$ & $0.10 \pm 0.01^{a}$ & $0.24 \pm 0.01$ & $0.18 \pm 0.01^{\mathrm{b}}$ & $0.17 \pm 0.01^{\mathrm{a}}$ & $0.24 \pm 0.01^{\mathrm{b}}$ & nd \\
\hline \multirow[t]{3}{*}{$p$-values } & Homoscedasticity ${ }^{2}$ & 0.025 & 0.004 & $<0.001$ & $<0.001$ & 0.034 & 0.828 & $<0.001$ & 0.005 & 0.001 & 0.001 & 0.003 & - \\
\hline & Normal distribution $^{3}$ & $<0.001$ & 0.117 & $<0.001$ & $<0.001$ & 0.005 & 0.547 & 0.037 & 0.277 & 0.024 & 0.002 & $<0.001$ & - \\
\hline & 1 -way ANOVA ${ }^{4}$ & $<0.001$ & $<0.001$ & $<0.001$ & $<0.001$ & $<0.001$ & $<0.001$ & $<0.001$ & 0.507 & $<0.001$ & $<0.001$ & $<0.001$ & - \\
\hline \multicolumn{14}{|c|}{ Mentha piperita } \\
\hline \multirow[t]{3}{*}{ GI } & 0 kGy & $0.15 \pm 0.02^{\mathrm{a}}$ & $1.0 \pm 0.1^{\mathrm{a}}$ & $0.12 \pm 0.01^{\mathrm{b}}$ & $0.14 \pm 0.01^{\mathrm{b}}$ & $0.15 \pm 0.01^{\mathrm{a}}$ & $0.59 \pm 0.05^{\mathrm{a}}$ & $0.04 \pm 0.01$ & $0.44 \pm 0.01^{\mathrm{b}}$ & $0.25 \pm 0.01^{\mathrm{b}}$ & $0.19 \pm 0.01^{\mathrm{a}}$ & $0.45 \pm 0.04^{\mathrm{b}}$ & $0.11 \pm 0.01^{c}$ \\
\hline & $1 \mathrm{kGy}$ & $0.16 \pm 0.02^{\mathrm{a}}$ & $1.0 \pm 0.1^{\mathrm{a}}$ & $0.17 \pm 0.02^{\mathrm{a}}$ & $0.15 \pm 0.02^{\mathrm{b}}$ & $0.12 \pm 0.01^{\mathrm{b}}$ & $0.48 \pm 0.01^{\mathrm{b}}$ & $0.05 \pm 0.01$ & $0.47 \pm 0.01^{\mathrm{a}}$ & $0.28 \pm 0.05^{\mathrm{b}}$ & $0.18 \pm 0.01^{\mathrm{b}}$ & $0.47 \pm 0.02^{\mathrm{b}}$ & $0.21 \pm 0.04$ \\
\hline & $10 \mathrm{kGy}$ & $0.10 \pm 0.03^{\mathrm{b}}$ & $0.9 \pm 0.1^{\mathrm{b}}$ & $0.11 \pm 0.01^{\mathrm{b}}$ & $0.20 \pm 0.01^{\mathrm{a}}$ & $0.09 \pm 0.01^{\mathrm{c}}$ & $0.53 \pm 0.04^{\mathrm{b}}$ & $0.04 \pm 0.01$ & $0.45 \pm 0.02^{\mathrm{b}}$ & $0.52 \pm 0.02^{\mathrm{a}}$ & $0.16 \pm 0.01^{\mathrm{c}}$ & $0.54 \pm 0.02^{\mathrm{a}}$ & $0.28 \pm 0.02^{\circ}$ \\
\hline \multirow[t]{3}{*}{$p$-values } & Homoscedasticity $^{2}$ & 0.437 & 0.002 & 0.021 & 0.992 & $<0.001$ & $<0.001$ & 0.260 & $<0.001$ & $<0.001$ & 0.207 & 0.036 & 0.016 \\
\hline & Normal distribution $^{3}$ & 0.118 & 0.022 & $<0.001$ & 0.035 & 0.011 & $<0.001$ & 0.218 & 0.084 & $<0.001$ & 0.885 & 0.604 & 0.006 \\
\hline & 1-way ANOVA ${ }^{4}$ & $<0.001$ & $<0.001$ & $<0.001$ & $<0.001$ & $<0.001$ & $<0.001$ & 0.135 & 0.001 & $<0.001$ & $<0.001$ & $<0.001$ & $<0.001$ \\
\hline
\end{tabular}




\subsubsection{Antioxidant activity}

DPPH radical-scavenging activity was evaluated by using an ELX800 microplate reader (Bio-Tek Instruments, Inc.; Winooski, VT, USA), and calculated as a percentage of DPPH discoloration using the formula: $\left[\left(A_{D P P H}-A_{S}\right) / A_{D P P H}\right] \times 100$, where $A_{S}$ is the absorbance of the solution containing the sample at $515 \mathrm{~nm}$, and $A_{\mathrm{DPPH}}$ is the absorbance of the DPPH solution. Reducing power was evaluated by the capacity to convert $\mathrm{Fe}^{3+}$ into $\mathrm{Fe}^{2+}$, measuring the absorbance at $690 \mathrm{~nm}$ in the microplate reader mentioned above. Inhibition of $\beta$-carotene bleaching was evaluated though the $\beta$-carotene/linoleate assay; the neutralization of linoleate free radicals avoids $\beta$-carotene bleaching, which is measured by the formula: $\beta$-carotene absorbance after $2 \mathrm{~h}$ of assay/initial absorbance) $\times 100 \%$ (Pereira et al., 2013).

\subsection{Statistical analysis}

For each irradiation dose and plant species, three independent samples were analyzed. Each of the samples was taken after pooling the plants treated in the same conditions together. Data were expressed as mean \pm standard deviation. All statistical tests were performed at a $5 \%$ significance level using IBM SPSS Statistics for Windows, version 22.0. (IBM Corp., USA).

The fulfillment of the one-way ANOVA requirements, specifically the normal distribution of the residuals and the homogeneity of variance, was tested by means of the Shapiro Wilk's and the Levene's tests, respectively. All dependent variables were compared using Tukey's honestly significant difference (HSD) or Tamhane's T2 multiple comparison tests, when homoscedasticity was verified or not, respectively.

Principal component analysis (PCA) was applied as a pattern recognition unsupervised classification method. The number of dimensions to keep for data analysis was assessed by the respective eigenvalues (which should be greater than one), by Cronbach's alpha parameter (that must be positive) and also by the total percentage of variance (that should be as high as possible) explained by the number of components selected. The number of plotted dimensions was chosen in order to allow meaningful interpretations.

\section{Results and discussion}

\subsection{Effects on chemical parameters}

The proximate composition and color parameters (Table 1) of $A$. citrodora (lemon verbena), M. officinalis (lemon balm), M. melissophyllum (bastard balm) and M. piperita (peppermint) showed some similarity, with carbohydrates as predominant component, followed by ash, protein and fat contents. Except for lemon balm, the proximate composition of these species is described for the first time. The nutritional profile detected for lemon balm is coherent to that reported in previous works (Dias, Barros, Sousa, \& Ferreira, 2012). Regarding the effect of gamma irradiation (GI), all these parameters showed to be relatively susceptible $(p<0.05)$, except ash content in lemon balm $(p=0.072)$. Despite the detected variations, it was not possible to identify overall tendencies, with the exception of protein content, which tended to be higher in samples irradiated with $10 \mathrm{kGy}$ for all species. The increase in protein content might be related to chemical processes (scission of the carbon-nitrogen bonds in the backbone of the polypeptide chain or splitting of the disulfide bonds) or to physical changes (like unfolding), which are commonly associated to irradiation treatment (Molins, 2001).

Color parameters are assessed in the quality control of post-harvest preservation processes (Hsu, Simonne, Jitareerat, \& Marshall, 2010). Herein, these parameters were also similar, with higher lightness values in lemon verbena $(\approx 49)$ and lemon balm $(\approx 49)$, lower redness in lemon verbena $(\approx-8.4)$ and bastard balm $(\approx-8.2)$ and higher yellowness $(\approx 27)$ in lemon verbena. Color parameters proved to be less susceptible to irradiation than those evaluated in the proximate analysis, since the detected differences had no statistical significance $(p>0.050)$ in most cases. Considering the cases where a statistically significant difference was found, it might be said that lightness, redness and yellowness leaned toward lower values in samples irradiated with $10 \mathrm{kGy}$. That is similar with the decrease of $\mathrm{a}^{*}$ and $\mathrm{b}^{*}$ observed in gamma irradiated green tea extracts (Jo, Son, Shin, \& Byun, 2003). The results for peppermint are in agreement with those reported in North American samples, showing no variation in color parameters when irradiated with low doses (Hsu et al., 2010).

Concerning free sugar composition (Table 2), fructose, glucose, sucrose and trehalose were quantified in all species. A fifth sugar was also quantified in bastard balm, but its identity could not be determined. Sucrose was the main sugar in lemon verbena $(\approx 6.7 \mathrm{~g} / 100 \mathrm{~g} \mathrm{dw})$ and lemon balm $(\approx 5.3 \mathrm{~g} / 100 \mathrm{~g} \mathrm{dw})$, while the unidentified sugar $(\approx 2.7 \mathrm{~g} / 100 \mathrm{~g} \mathrm{dw})$ and trehalose $(\approx 0.9 \mathrm{~g} / 100 \mathrm{~g} \mathrm{dw})$ were the most abundant in bastard balm and peppermint, respectively. Lemon verbena showed the highest content $(\approx 10.2 \mathrm{~g} / 100 \mathrm{~g} \mathrm{dw})$ in total sugars. The $10 \mathrm{kGy}$ dose seemed to increase sugars content in lemon balm and bastard balm, while lemon verbena and peppermint tended to present higher values in unirradiated samples. The increase in free sugars, which was previously reported in soybean (Byun, Kang, \& Mori, 1996), ginseng (Byun, Yook, Kwon, \& Kang, 1997), green, black and oolong teas (Kausar, Akram, \& Kwon, 2013) and plan waste materials (Tissot, Grdanovska, Barkatt, Silverman, \& Al-Sheikhly, 2013) as a result of gamma irradiation, might be explained by the shortening or depolymerization of polysaccharide molecules. Other verified changes might be explained by variations in the optical rotation of sugars, which is a common occurrence under irradiation treatment (Molins, 2001).

Peppermint gave the highest content in organic acids (Table 2), mainly due to the citric acid amounts $(\approx 7.6 \mathrm{~g} / 100 \mathrm{~g} \mathrm{dw})$. Malic acid $(\approx 5.5 \mathrm{~g} / 100 \mathrm{~g} \mathrm{dw})$ was the predominant form in bastard balm, while shikimic acid $(\approx 4.1 \mathrm{~g} / 100 \mathrm{~g} \mathrm{dw})$ and citric acid $(\approx 1.7 \mathrm{~g} / 100 \mathrm{~g} \mathrm{dw})$ were the organic acids quantified in highest amounts in lemon balm and lemon verbena, respectively. Oxalic acid and quinic acid (except in lemon verbena) were also quantified. In general, the highest changes were detected in samples irradiated with $1 \mathrm{kGy}$ dose, indicating that some degradation processes commonly triggered by the molecular oxygen inside the polyethylene bag might decrease due to an oxygen ionizing effect produced when using the $10 \mathrm{kGy}$ dose.

The four tocopherol isoforms $(\alpha, \beta, \gamma$ and $\delta$ ) were detected in all species, except for $\delta$-tocopherol in lemon verbena (Table 3 ). $\alpha$-Tocopherol was the main isoform in lemon balm $(\approx 30.3 \mathrm{mg} / 100 \mathrm{~g} \mathrm{dw})$, lemon verbena $(\approx 15.4 \mathrm{mg} / 100 \mathrm{~g} \mathrm{dw})$ and peppermint $(\approx 15.1 \mathrm{mg} / 100 \mathrm{~g} \mathrm{dw})$, while $\beta$-tocopherol predominated in bastard balm $(\approx 18.5 \mathrm{mg} / 100 \mathrm{~g}$ $\mathrm{dw}$ ). In line with previous results (Taipina, Lamardo, Rodas, \& Mastro, 2009), the tocopherol contents were significantly changed in response to irradiation treatment (especially for the $1 \mathrm{kGy}$ dose) in all the assayed samples, except for $\gamma$-tocopherol in peppermint $(p=0.797)$. These differences are mainly linked to $\alpha$-and $\beta$-tocopherol contents, which are not as stable to irradiation as $\gamma$-tocopherol, and are also recognized as having higher oxidative stability (Warner, Miller, \& Demurin, 2008).

Tables $4 \mathrm{~A}$ and $4 \mathrm{~B}$ present the individual fatty acids (FA) divided as those quantified below $1 \%$ in all species (Table $4 A$ ) and those quantified above $1 \%$ at least in one species (Table $4 \mathrm{~B}$ ). The predominant FA in the four species were linolenic acid (C18:3n3), followed by palmitic (C16:0) and linoleic (C18:2n6) acids in lemon verbena and lemon balm, linoleic and palmitic acids in bastard balm, and arachidic and palmitic acids in peppermint. The FA profile detected for lemon balm is similar to that reported previously in the same species (Dias et al., 2012). Despite the individual differences, polyunsaturated fatty acids (PUFA) were predominant in all species ( 52.6 to $69.5 \%$ ), followed by saturated fatty acids (SFA, 28.1 to $41.2 \%$ ) and monounsaturated fatty acids (MUFA, 2.07 to 16.6\%) (Table 4B). The detected percentages were significantly changed by irradiation treatment with the exceptions of C23:0 in lemon balm ( $p=0.110), \mathrm{C} 17: 0(p=0.507), \mathrm{C} 24: 0(p=0.124)$ and SFA $(p=0.214)$ in bastard balm and C15:1 $(p=0.135)$ and $\mathrm{C16:0}(p=$ 
Table 4B

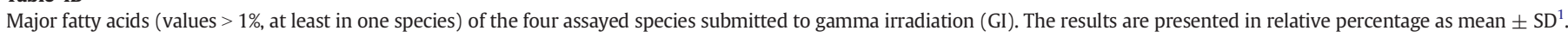

\begin{tabular}{|c|c|c|c|c|c|c|c|c|c|}
\hline & & C10:0 & $\mathrm{C} 14: 0$ & C14:1 & C16:0 & C16:1 & C18:0 & C18:1n9 & C18:2n6 \\
\hline \multicolumn{10}{|c|}{ Aloysia citrodora } \\
\hline \multirow[t]{3}{*}{ GI } & $0 \mathrm{kGy}$ & nd & $1.1 \pm 0.1^{\mathrm{b}}$ & nd & $15.7 \pm 0.2^{\mathrm{b}}$ & $0.50 \pm 0.02^{\mathrm{b}}$ & $1.17 \pm 0.01^{\mathrm{b}}$ & $0.95 \pm 0.02^{\mathrm{b}}$ & $12.6 \pm 0.1^{\mathrm{a}}$ \\
\hline & $1 \mathrm{kGy}$ & nd & $1.3 \pm 0.1^{\mathrm{a}}$ & nd & $15.8 \pm 0.4^{\mathrm{b}}$ & $0.62 \pm 0.01^{a}$ & $1.10 \pm 0.01^{\mathrm{c}}$ & $0.95 \pm 0.02^{\mathrm{b}}$ & $12.4 \pm 0.1^{\mathrm{b}}$ \\
\hline & $10 \mathrm{kGy}$ & nd & $0.9 \pm 0.1^{\mathrm{c}}$ & nd & $16.6 \pm 0.5^{\mathrm{a}}$ & $0.64 \pm 0.03^{\mathrm{a}}$ & $1.31 \pm 0.01^{\mathrm{a}}$ & $1.13 \pm 0.03^{\mathrm{a}}$ & $12.6 \pm 0.1^{\mathrm{a}}$ \\
\hline \multirow[t]{3}{*}{$p$-values } & Homoscedasticity $^{2}$ & - & 0.273 & - & 0.071 & 0.008 & 0.002 & 0.225 & $<0.001$ \\
\hline & Normal distribution $^{3}$ & - & 0.080 & - & 0.025 & 0.001 & $<0.001$ & $<0.001$ & $<0.001$ \\
\hline & 1-way ANOVA ${ }^{4}$ & - & $<0.001$ & - & $<0.001$ & $<0.001$ & $<0.001$ & $<0.001$ & $<0.001$ \\
\hline \multicolumn{10}{|c|}{ Melissa officinalis } \\
\hline \multirow[t]{3}{*}{ GI } & $0 \mathrm{kGy}$ & $0.29 \pm 0.02^{\mathrm{a}}$ & $2.9 \pm 0.1^{\mathrm{a}}$ & $0.53 \pm 0.01^{\mathrm{b}}$ & $22.7 \pm 0.3^{\mathrm{a}}$ & nd & $3.6 \pm 0.1^{\mathrm{a}}$ & $4.9 \pm 0.2^{\mathrm{a}}$ & $15.3 \pm 0.4^{\mathrm{ab}}$ \\
\hline & $1 \mathrm{kGy}$ & $0.25 \pm 0.01^{\mathrm{b}}$ & $2.6 \pm 0.1^{\mathrm{b}}$ & $0.52 \pm 0.01^{\mathrm{b}}$ & $20.9 \pm 0.1^{\mathrm{c}}$ & nd & $3.6 \pm 0.1^{\mathrm{a}}$ & $4.8 \pm 0.1^{\mathrm{a}}$ & $15.0 \pm 0.1^{\mathrm{b}}$ \\
\hline & $10 \mathrm{kGy}$ & $0.22 \pm 0.01^{\mathrm{c}}$ & $2.4 \pm 0.1^{c}$ & $0.62 \pm 0.02^{\mathrm{a}}$ & $21.5 \pm 0.1^{\mathrm{b}}$ & nd & $3.2 \pm 0.1^{\mathrm{b}}$ & $4.3 \pm 0.1^{b}$ & $15.5 \pm 0.1^{\mathrm{a}}$ \\
\hline \multirow[t]{3}{*}{$p$-values } & Homoscedasticity $^{2}$ & 0.001 & $<0.001$ & $<0.001$ & $<0.001$ & - & 0.048 & $<0.001$ & $<0.001$ \\
\hline & Normal distribution $^{3}$ & 0.061 & 0.002 & $<0.001$ & 0.002 & - & 0.002 & 0.001 & 0.062 \\
\hline & 1-way ANOVA ${ }^{4}$ & $<0.001$ & $<0.001$ & $<0.001$ & $<0.001$ & - & $<0.001$ & $<0.001$ & 0.001 \\
\hline \multicolumn{10}{|c|}{ Melittis melissophyllum } \\
\hline \multirow[t]{3}{*}{ GI } & $0 \mathrm{kGy}$ & nd & $0.58 \pm 0.03^{c}$ & nd & $14.3 \pm 0.2^{\mathrm{b}}$ & $1.29 \pm 0.05^{\mathrm{a}}$ & $2.41 \pm 0.05^{\mathrm{b}}$ & $11.5 \pm 0.3^{c}$ & $14.8 \pm 0.4^{\mathrm{c}}$ \\
\hline & $1 \mathrm{kGy}$ & nd & $0.81 \pm 0.05^{\mathrm{b}}$ & nd & $14.2 \pm 0.5^{\mathrm{b}}$ & $1.14 \pm 0.03^{b}$ & $2.43 \pm 0.01^{\mathrm{b}}$ & $13.0 \pm 0.4^{\mathrm{b}}$ & $16.2 \pm 0.4^{\mathrm{b}}$ \\
\hline & $10 \mathrm{kGy}$ & nd & $0.92 \pm 0.03^{\mathrm{a}}$ & nd & $15.1 \pm 0.1^{\mathrm{a}}$ & $1.25 \pm 0.04^{\mathrm{a}}$ & $2.76 \pm 0.01^{\mathrm{a}}$ & $15.1 \pm 0.5^{\mathrm{a}}$ & $18.2 \pm 0.4^{\mathrm{a}}$ \\
\hline \multirow[t]{3}{*}{$p$-values } & Homoscedasticity $^{2}$ & - & 0.022 & - & $<0.001$ & 0.005 & 0.004 & $<0.001$ & 0.964 \\
\hline & Normal distribution $^{3}$ & - & 0.004 & - & 0.006 & 0.214 & $<0.001$ & 0.029 & 0.049 \\
\hline & 1-way ANOVA ${ }^{4}$ & - & $<0.001$ & - & $<0.001$ & $<0.001$ & $<0.001$ & $<0.001$ & $<0.001$ \\
\hline \multicolumn{10}{|c|}{ Mentha piperita } \\
\hline \multirow[t]{3}{*}{ GI } & $0 \mathrm{kGy}$ & $0.07 \pm 0.01^{\mathrm{a}}$ & $1.4 \pm 0.1^{\mathrm{b}}$ & $1.2 \pm 0.1^{\mathrm{a}}$ & $10.4 \pm 0.3$ & $0.88 \pm 0.05^{\mathrm{b}}$ & $2.47 \pm 0.03^{b}$ & $1.62 \pm 0.05^{\mathrm{b}}$ & $7.3 \pm 0.1^{\mathrm{b}}$ \\
\hline & $1 \mathrm{kGy}$ & $0.04 \pm 0.01^{\mathrm{b}}$ & $1.5 \pm 0.1^{\mathrm{a}}$ & $1.2 \pm 0.1^{\mathrm{a}}$ & $10.4 \pm 0.3$ & $0.97 \pm 0.01^{\mathrm{a}}$ & $2.55 \pm 0.01^{\mathrm{a}}$ & $1.61 \pm 0.01^{\mathrm{b}}$ & $7.5 \pm 0.1^{\mathrm{a}}$ \\
\hline & $10 \mathrm{kGy}$ & $0.02 \pm 0.01^{\mathrm{c}}$ & $1.6 \pm 0.1^{\mathrm{a}}$ & $1.0 \pm 0.1^{\mathrm{b}}$ & $10.1 \pm 0.5$ & $0.81 \pm 0.05^{\mathrm{b}}$ & $2.60 \pm 0.05^{\mathrm{a}}$ & $1.91 \pm 0.05^{\mathrm{a}}$ & $7.2 \pm 0.1^{c}$ \\
\hline \multirow[t]{3}{*}{$p$-values } & Homoscedasticity $^{2}$ & 0.160 & 0.062 & 0.001 & 0.036 & 0.001 & 0.005 & 0.001 & 0.001 \\
\hline & Normal distribution $^{3}$ & 0.008 & 0.660 & 0.179 & 0.103 & 0.017 & 0.509 & $<0.001$ & 0.006 \\
\hline & 1-way ANOVA ${ }^{4}$ & $<0.001$ & $<0.001$ & $<0.001$ & 0.313 & $<0.001$ & $<0.001$ & $<0.001$ & $<0.001$ \\
\hline
\end{tabular}

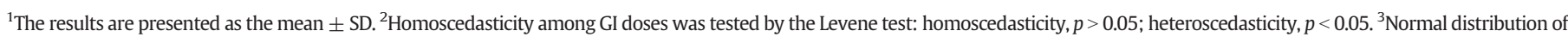

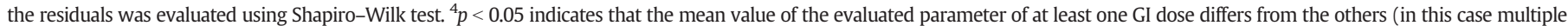
comparison tests were performed). For each species, means within a column with different letters differ significantly $(p<0.05)$.

0.313 ) in peppermint. The differences verified for irradiated samples might be explained by mechanisms of lipid radiolysis, involving primary ionization, followed by migration of the positive charge either toward the carboxyl carbonyl group or double bonds (Molins, 2001).

\subsection{Effects on antioxidant parameters}

In order to compare the effects of gamma irradiation on the antioxidant activity, three in vitro assays were applied: scavenging effects on DPPH radicals (measures the decrease in DPPH radical absorption after exposure to radical scavengers), reducing power (conversion of a $\mathrm{Fe}^{3+}$ /ferricyanide complex to $\mathrm{Fe}^{2+}$ ) and inhibition of $\beta$-carotene bleaching (measures the capacity to neutralize the linoleate-free radical and other free radicals formed in the system which attack the highly unsaturated $\beta$-carotene models). Moreover, a preliminary quantification of total phenols and flavonoids subgroup was also performed; the results are expressed in Table 5. Among the assayed species, lemon balm showed the highest antioxidant activity on all the assays, especially concerning the infusions, presenting values similar to those published in Iranian (Dastmalchi et al., 2008) and Brazillian (Kamdem et al., 2013) samples. The $\mathrm{EC}_{50}$ values are close to those reported in previous studies. Nevertheless, the infusions prepared in this study gave lower amounts of bioactive compounds (Dias et al., 2012). On the other hand, bastard balm proved to be the least effective in terms of antioxidant activity, as well as phenols and flavonoids content. The methanolic extracts gave higher activities than the corresponding infusions, showing to be correlated with the amounts of bioactive compounds quantified in each case.

Changes induced by gamma irradiation proved to be statistically significant in almost all cases, except for DPPH scavenging activity in methanolic extracts $(p=0.996)$ of bastard balm. Likewise, changes in bioactive compound amounts were always significant except for phenols content in the infusions of bastard balm ( $p=0.474)$. Despite the significant changes found within these parameters, it was not possible to identify unequivocal tendencies common to all assays and/or plant species.

\subsection{Principal component analysis (PCA)}

In the former section, the differences resulting from gamma irradiation were compared considering the individual effect within each species. Despite the high number of statistically significant changes, it was not possible to identify overall trends, which might characterize the effects of gamma irradiation. Furthermore, it was intended to validate this technology independently of the treated plant species. Accordingly, in the present section the results were evaluated considering data for all species and parameters simultaneously.

Hence, to verify if irradiation maintains the chemical profile, principal component analysis (PCA) was applied. In this analysis, instead of evaluating individual changes caused in each parameter, the effects in all parameters were considered at once. Due to the great variation (in some parameters) among species, the values were normalized by subtracting the value corresponding to unirradiated samples to those from 1 and $10 \mathrm{kGy}$ irradiations. The obtained differences were further divided by the value of the respective control. In this way, the classification procedure was applied to the differences caused by irradiation and not to the absolute values measured for each parameter. Due to practical reasons, only the parameters detected in the four species were included in this study.

The plot of object scores (Fig. 1A) for gamma irradiation dose, indicated that the first two dimensions (first: Cronbach's $\alpha, 0.941$; eigenvalue, 13.031; second: Cronbach's $\alpha, 0.915$; eigenvalue, 9.819) account for most of the variance of all quantified variables $(34.1 \%$ and $28.1 \%$, respectively). The included variance would ideally be higher, 


\begin{tabular}{|c|c|c|c|c|c|c|c|c|c|c|}
\hline C18:3n6 & C18:3n3 & C20:0 & $C 20: 5 n 3$ & $\mathrm{C} 22: 0$ & C23:0 & C22:6n3 & $\mathrm{C} 24: 0$ & SFA & MUFA & PUFA \\
\hline \multicolumn{11}{|c|}{ Aloysia citrodora } \\
\hline nd & $56.2 \pm 0.3^{\mathrm{a}}$ & $0.87 \pm 0.02^{\mathrm{b}}$ & nd & $1.00 \pm 0.02^{\mathrm{a}}$ & $5.4 \pm 0.1^{b}$ & nd & $1.4 \pm 0.1^{c}$ & $28.6 \pm 0.2^{\mathrm{b}}$ & $2.07 \pm 0.03^{c}$ & $69.3 \pm 0.3^{\mathrm{a}}$ \\
\hline nd & $56.6 \pm 0.5^{\mathrm{a}}$ & $0.99 \pm 0.03^{\mathrm{a}}$ & nd & $0.82 \pm 0.01^{\mathrm{c}}$ & $4.2 \pm 0.1^{c}$ & nd & $1.7 \pm 0.1^{\mathrm{b}}$ & $28.1 \pm 0.5^{c}$ & $2.42 \pm 0.03^{\mathrm{a}}$ & $69.5 \pm 0.5^{\mathrm{a}}$ \\
\hline nd & $54.3 \pm 0.4^{\mathrm{b}}$ & $0.59 \pm 0.04^{c}$ & nd & $0.93 \pm 0.04^{\mathrm{b}}$ & $5.9 \pm 0.4^{\mathrm{a}}$ & nd & $1.8 \pm 0.1^{\mathrm{a}}$ & $30.3 \pm 0.5^{\mathrm{a}}$ & $2.27 \pm 0.03^{b}$ & $67.4 \pm 0.5^{\mathrm{b}}$ \\
\hline- & 0.259 & 0.265 & - & 0.001 & $<0.001$ & - & $<0.001$ & 0.158 & 0.742 & 0.231 \\
\hline- & 0.007 & 0.001 & - & 0.004 & 0.001 & - & 0.003 & 0.045 & 0.033 & 0.005 \\
\hline- & $<0.001$ & $<0.001$ & - & $<0.001$ & $<0.001$ & - & $<0.001$ & $<0.001$ & $<0.001$ & $<0.001$ \\
\hline \multicolumn{11}{|c|}{ Melissa officinalis } \\
\hline nd & $33.2 \pm 0.5^{c}$ & $3.4 \pm 0.1^{\mathrm{c}}$ & $3.9 \pm 0.1^{\mathrm{b}}$ & $1.3 \pm 0.1^{\mathrm{b}}$ & $3.3 \pm 0.2$ & nd & $1.2 \pm 0.2^{\mathrm{ab}}$ & $41.2 \pm 0.5^{\mathrm{a}}$ & $6.2 \pm 0.2^{\mathrm{a}}$ & $52.6 \pm 0.5^{c}$ \\
\hline nd & $34.4 \pm 0.1^{\mathrm{b}}$ & $3.9 \pm 0.1^{\mathrm{a}}$ & $4.5 \pm 0.1^{\mathrm{a}}$ & $1.5 \pm 0.1^{\mathrm{a}}$ & $3.2 \pm 0.1$ & nd & $1.3 \pm 0.1^{\mathrm{a}}$ & $39.7 \pm 0.2^{\mathrm{b}}$ & $6.0 \pm 0.1^{b}$ & $54.3 \pm 0.1^{\mathrm{b}}$ \\
\hline nd & $36.3 \pm 0.2^{\mathrm{a}}$ & $3.5 \pm 0.1^{b}$ & $3.5 \pm 0.1^{\mathrm{c}}$ & $1.5 \pm 0.1^{\mathrm{a}}$ & $3.1 \pm 0.1$ & nd & $1.1 \pm 0.1^{\mathrm{b}}$ & $38.7 \pm 0.2^{\mathrm{c}}$ & $5.6 \pm 0.1^{c}$ & $55.7 \pm 0.2^{\mathrm{a}}$ \\
\hline- & 0.003 & 0.002 & 0.437 & $<0.001$ & 0.005 & - & 0.107 & 0.005 & $<0.001$ & 0.007 \\
\hline- & 0.012 & $<0.001$ & 0.002 & $<0.001$ & 0.033 & - & 0.411 & 0.041 & 0.020 & 0.029 \\
\hline- & $<0.001$ & $<0.001$ & $<0.001$ & $<0.001$ & 0.110 & - & 0.004 & $<0.001$ & $<0.001$ & $<0.001$ \\
\hline \multicolumn{11}{|c|}{ Melittis melissophyllum } \\
\hline $5.8 \pm 0.1^{b}$ & $36 \pm 1^{a}$ & $0.88 \pm 0.02^{\mathrm{b}}$ & nd & $1.3 \pm 0.1^{\mathrm{b}}$ & $6.2 \pm 0.2^{\mathrm{a}}$ & nd & $3.0 \pm 0.1$ & $30.4 \pm 0.2$ & $13.1 \pm 0.2^{c}$ & $56.5 \pm 0.2^{\mathrm{a}}$ \\
\hline $5.8 \pm 0.1^{b}$ & $33 \pm 1^{\mathrm{b}}$ & $0.96 \pm 0.02^{\mathrm{a}}$ & nd & $1.3 \pm 0.1^{\mathrm{b}}$ & $5.9 \pm 0.4^{\mathrm{a}}$ & nd & $2.9 \pm 0.2$ & $30.1 \pm 0.4$ & $14.4 \pm 0.3^{\mathrm{b}}$ & $55.5 \pm 0.5^{b}$ \\
\hline $6.3 \pm 0.1^{a}$ & $28 \pm 1^{c}$ & $0.97 \pm 0.03^{\mathrm{a}}$ & nd & $1.4 \pm 0.1^{\mathrm{a}}$ & $4.1 \pm 0.1^{b}$ & nd & $3.1 \pm 0.2$ & $30.2 \pm 0.3$ & $16.6 \pm 0.5^{\mathrm{a}}$ & $53.2 \pm 0.5^{c}$ \\
\hline 0.009 & 0.010 & 0.497 & - & $<0.001$ & $<0.001$ & - & 0.002 & 0.186 & $<0.001$ & 0.001 \\
\hline$<0.001$ & 0.003 & 0.454 & - & 0.001 & $<0.001$ & - & 0.491 & 0.532 & 0.013 & 0.005 \\
\hline$<0.001$ & $<0.001$ & $<0.001$ & - & 0.003 & $<0.001$ & - & 0.124 & 0.214 & $<0.001$ & $<0.001$ \\
\hline \multicolumn{11}{|c|}{ Mentha piperita } \\
\hline nd & $46 \pm 1^{a}$ & $15.8 \pm 0.5^{c}$ & $2.8 \pm 0.2^{c}$ & $2.6 \pm 0.1^{\mathrm{b}}$ & $0.24 \pm 0.0^{\mathrm{b}}$ & $1.4 \pm 0.1^{\mathrm{c}}$ & $2.1 \pm 0.1^{\mathrm{a}}$ & $38 \pm 1^{\mathrm{c}}$ & $4.1 \pm 0.1^{c}$ & $58 \pm 1^{\mathrm{a}}$ \\
\hline nd & $44 \pm 1^{\mathrm{b}}$ & $16.7 \pm 0.5^{\mathrm{b}}$ & $3.0 \pm 0.1^{\mathrm{b}}$ & $2.8 \pm 0.1^{\mathrm{a}}$ & $0.21 \pm 0.0^{c}$ & $1.5 \pm 0.1^{\mathrm{a}}$ & $1.9 \pm 0.1^{\mathrm{b}}$ & $39 \pm 1^{\mathrm{b}}$ & $4.3 \pm 0.1^{b}$ & $57 \pm 1^{\mathrm{b}}$ \\
\hline nd & $43 \pm 1^{\mathrm{c}}$ & $17.9 \pm 0.1^{\mathrm{a}}$ & $3.3 \pm 0.1^{\mathrm{a}}$ & $2.9 \pm 0.1^{\mathrm{a}}$ & $0.26 \pm 0.0^{\mathrm{a}}$ & $1.6 \pm 0.1^{\mathrm{a}}$ & $1.9 \pm 0.1^{\mathrm{b}}$ & $40 \pm 1^{\mathrm{a}}$ & $4.6 \pm 0.2^{\mathrm{a}}$ & $56 \pm 1^{c}$ \\
\hline- & 0.151 & 0.001 & $<0.001$ & 0.237 & $<0.001$ & $<0.001$ & 0.058 & 0.134 & 0.361 & 0.050 \\
\hline- & 0.246 & 0.012 & 0.057 & 0.904 & 0.002 & $<0.001$ & 0.262 & 0.381 & 0.815 & 0.247 \\
\hline - & $<0.001$ & $<0.001$ & $<0.001$ & 0.001 & $<0.001$ & $<0.001$ & $<0.001$ & $<0.001$ & $<0.001$ & $<0.001$ \\
\hline
\end{tabular}

Table 5

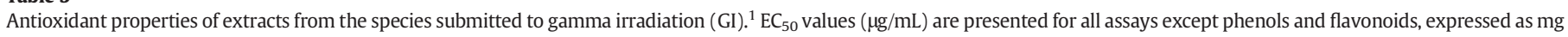
$\mathrm{GAE} / \mathrm{g}$ extract and $\mathrm{mg} \mathrm{CE} / \mathrm{g}$ extract, respectively.

\begin{tabular}{|c|c|c|c|c|c|c|c|c|c|c|c|}
\hline & & \multicolumn{2}{|c|}{$\begin{array}{l}\text { DPPH scavenging } \\
\text { activity }\end{array}$} & \multicolumn{2}{|c|}{ Reducing power } & \multicolumn{2}{|c|}{$\begin{array}{l}\beta \text {-carotene bleaching } \\
\text { inhibition }\end{array}$} & \multicolumn{2}{|l|}{ Phenols } & \multicolumn{2}{|l|}{ Flavonoids } \\
\hline & & Infusion & $\mathrm{MeOH}$ & Infusion & $\mathrm{MeOH}$ & Infusion & $\mathrm{MeOH}$ & Infusion & $\mathrm{MeOH}$ & Infusion & $\mathrm{MeOH}$ \\
\hline \multicolumn{12}{|c|}{ Aloysia citrodora } \\
\hline \multirow[t]{3}{*}{ GI } & $0 \mathrm{kGy}$ & $232 \pm 8^{a}$ & $39 \pm 4^{c}$ & $169 \pm 1^{\mathrm{b}}$ & $22.8 \pm 0.3^{\mathrm{c}}$ & $580 \pm 31^{\mathrm{c}}$ & $208 \pm 9^{b}$ & $134 \pm 8^{\mathrm{c}}$ & $665 \pm 13^{\mathrm{a}}$ & $92 \pm 1^{\mathrm{a}}$ & $369 \pm 5^{a}$ \\
\hline & 1 kGy & $237 \pm 5^{a}$ & $90 \pm 6^{\mathrm{b}}$ & $184 \pm 2^{a}$ & $49.2 \pm 0.4^{\mathrm{b}}$ & $1004 \pm 23^{\mathrm{a}}$ & $235 \pm 5^{a}$ & $188 \pm 2^{\mathrm{b}}$ & $531 \pm 34^{\mathrm{b}}$ & $60 \pm 2^{c}$ & $359 \pm 9^{b}$ \\
\hline & $10 \mathrm{kGy}$ & $205 \pm 16^{\mathrm{b}}$ & $109 \pm 4^{\mathrm{a}}$ & $170 \pm 1^{\mathrm{b}}$ & $62 \pm 1^{\mathrm{a}}$ & $829 \pm 36^{\mathrm{b}}$ & $198 \pm 6^{c}$ & $205 \pm 3^{\mathrm{a}}$ & $455 \pm 12^{\mathrm{c}}$ & $76 \pm 3^{b}$ & $277 \pm 2^{c}$ \\
\hline \multirow[t]{3}{*}{$p$-values } & Homoscedasticity $^{\mathrm{b}}$ & 0.002 & 0.238 & 0.031 & 0.005 & 0.340 & 0.200 & 0.002 & $<0.001$ & $<0.001$ & $<0.001$ \\
\hline & Normal distribution $^{c}$ & 0.002 & $<0.001$ & $<0.001$ & $<0.001$ & 0.005 & 0.033 & $<0.001$ & 0.002 & 0.001 & $<0.001$ \\
\hline & 1-way ANOVA & $<0.001$ & $<0.001$ & $<0.001$ & $<0.001$ & $<0.001$ & $<0.001$ & $<0.001$ & $<0.001$ & $<0.001$ & $<0.001$ \\
\hline \multicolumn{12}{|c|}{ Melissa officinalis } \\
\hline \multirow[t]{3}{*}{ GI } & $0 \mathrm{kGy}$ & $101 \pm 3^{\mathrm{b}}$ & $67 \pm 1^{\mathrm{b}}$ & $80 \pm 1^{b}$ & $44 \pm 1^{\mathrm{c}}$ & $165 \pm 4^{\mathrm{a}}$ & $125 \pm 3^{a}$ & $100 \pm 1^{\mathrm{c}}$ & $829 \pm 6^{a}$ & $63 \pm 1^{c}$ & $448 \pm 4^{\mathrm{b}}$ \\
\hline & 1 kGy & $101 \pm 1^{\mathrm{b}}$ & $73 \pm 3^{a}$ & $75 \pm 1^{\mathrm{c}}$ & $48 \pm 1^{b}$ & $130 \pm 5^{c}$ & $113 \pm 2^{b}$ & $108 \pm 2^{\mathrm{a}}$ & $786 \pm 22^{\mathrm{b}}$ & $69 \pm 1^{\mathrm{a}}$ & $498 \pm 11^{\mathrm{a}}$ \\
\hline & 10 kGy & $107 \pm 2^{\mathrm{a}}$ & $73 \pm 2^{a}$ & $103 \pm 1^{\mathrm{a}}$ & $55 \pm 1^{\mathrm{a}}$ & $135 \pm 2^{\mathrm{b}}$ & $109 \pm 2^{c}$ & $104 \pm 2^{\mathrm{b}}$ & $742 \pm 8^{c}$ & $65 \pm 1^{\mathrm{b}}$ & $417 \pm 4^{\mathrm{c}}$ \\
\hline \multirow[t]{3}{*}{$p$-values } & Homoscedasticity $^{\mathrm{b}}$ & $<0.001$ & 0.010 & 0.037 & 0.397 & 0.028 & 0.224 & $<0.001$ & $<0.001$ & $<0.001$ & 0.023 \\
\hline & Normal distribution $^{c}$ & 0.097 & 0.029 & $<0.001$ & $<0.001$ & $<0.001$ & 0.008 & 0.029 & 0.002 & 0.016 & 0.006 \\
\hline & 1-way ANOVA ${ }^{d}$ & $<0.001$ & $<0.001$ & $<0.001$ & $<0.001$ & $<0.001$ & $<0.001$ & $<0.001$ & $<0.001$ & $<0.001$ & $<0.001$ \\
\hline \multicolumn{12}{|c|}{ Melittis melissophyllum } \\
\hline \multirow[t]{3}{*}{ GI } & $0 \mathrm{kGy}$ & $583 \pm 24^{\mathrm{c}}$ & $354 \pm 39$ & $512 \pm 16^{\mathrm{b}}$ & $249 \pm 2^{\mathrm{b}}$ & $1648 \pm 154^{\mathrm{c}}$ & $447 \pm 66^{\mathrm{b}}$ & $70 \pm 4$ & $160 \pm 3^{\mathrm{a}}$ & $29 \pm 2^{\mathrm{a}}$ & $108 \pm 4^{\mathrm{a}}$ \\
\hline & $1 \mathrm{kGy}$ & $696 \pm 92^{b}$ & $355 \pm 19$ & $605 \pm 29^{a}$ & $198 \pm 3^{c}$ & $2105 \pm 139^{b}$ & $538 \pm 61^{a}$ & $73 \pm 5$ & $100 \pm 3^{c}$ & $16 \pm 1^{b}$ & $73 \pm 1^{c}$ \\
\hline & 10 kGy & $843 \pm 28^{a}$ & $354 \pm 23$ & $457 \pm 12^{\mathrm{c}}$ & $290 \pm 2^{\mathrm{a}}$ & $2299 \pm 187^{\mathrm{a}}$ & $595 \pm 37^{\mathrm{a}}$ & $70 \pm 3$ & $135 \pm 2^{\mathrm{b}}$ & $15 \pm 1^{\mathrm{b}}$ & $83 \pm 5^{b}$ \\
\hline \multirow[t]{3}{*}{$p$-values } & Homoscedasticity $^{\mathrm{b}}$ & 0.171 & 0.005 & 0.017 & 0.300 & 0.359 & 0.082 & 0.233 & 0.199 & $<0.001$ & $<0.001$ \\
\hline & Normal distribution $^{\mathrm{c}}$ & 0.008 & 0.007 & 0.054 & 0.001 & 0.286 & 0.060 & 0.007 & 0.001 & $<0.001$ & $<0.001$ \\
\hline & 1-way ANOVA & $<0.001$ & 0.996 & $<0.001$ & $<0.001$ & $<0.001$ & $<0.001$ & 0.474 & $<0.001$ & $<0.001$ & $<0.001$ \\
\hline \multicolumn{12}{|c|}{ Mentha piperita } \\
\hline \multirow[t]{3}{*}{ GI } & $0 \mathrm{kGy}$ & $184 \pm 5^{\mathrm{b}}$ & $83 \pm 7^{b}$ & $119 \pm 2^{\mathrm{c}}$ & $52 \pm 2^{\mathrm{a}}$ & $597 \pm 44^{\mathrm{b}}$ & $184 \pm 5^{\mathrm{a}}$ & $218 \pm 2^{c}$ & $591 \pm 19^{\mathrm{a}}$ & $117 \pm 2^{\mathrm{a}}$ & $319 \pm 6^{\mathrm{b}}$ \\
\hline & $1 \mathrm{kGy}$ & $192 \pm 6^{\mathrm{b}}$ & $98 \pm 5^{a}$ & $136 \pm 2^{\mathrm{b}}$ & $43 \pm 1^{b}$ & $465 \pm 5^{c}$ & $137 \pm 2^{\mathrm{b}}$ & $276 \pm 4^{\mathrm{a}}$ & $572 \pm 25^{\mathrm{a}}$ & $95 \pm 3^{b}$ & $354 \pm 3^{a}$ \\
\hline & 10 kGy & $225 \pm 9^{a}$ & $86 \pm 3^{b}$ & $146 \pm 4^{\mathrm{a}}$ & $53 \pm 1^{a}$ & $715 \pm 67^{\mathrm{a}}$ & $95 \pm 4^{c}$ & $242 \pm 4^{\mathrm{b}}$ & $527 \pm 13^{b}$ & $78 \pm 2^{\mathrm{c}}$ & $266 \pm 8^{c}$ \\
\hline \multirow[t]{3}{*}{$p$-values } & Homoscedasticity $^{\mathrm{b}}$ & 0.039 & 0.055 & 0.007 & $<0.001$ & $<0.001$ & 0.048 & 0.006 & 0.032 & 0.114 & 0.001 \\
\hline & Normal distribution $^{\mathrm{c}}$ & 0.002 & 0.316 & 0.002 & $<0.001$ & 0.009 & 0.002 & 0.001 & 0.018 & 0.002 & $<0.001$ \\
\hline & 1-way ANOVA ${ }^{\mathrm{d}}$ & $<0.001$ & $<0.001$ & $<0.001$ & $<0.001$ & $<0.001$ & $<0.001$ & $<0.001$ & $<0.001$ & $<0.001$ & $<0.001$ \\
\hline
\end{tabular}

MeOH-methanol; GAE-gallic acid equivalents; $\mathrm{CE}$-catechin equivalents.

a The results are presented as the mean $\pm \mathrm{SD}$.

b Homoscedasticity among GI doses was tested by the Levene test: homoscedasticity, $p>0.05$; heteroscedasticity, $p<0.05$.

c Normal distribution of the residuals was evaluated using Shapiro-Wilk test.

d $p<0.05$ indicates that the mean value of the evaluated parameter of at least one GI dose differs from the others (in this case multiple comparison tests were performed). For each species, means within a column with different letters differ significantly $(p<0.05)$. 
to each gamma irradiation dose ( $0 \mathrm{kGy}, 1 \mathrm{kGy}$ and $10 \mathrm{kGy}$ ) were not shaped, as it could have been anticipated from Tables 1-5. In fact, and as it can be concluded by comparing the plots of object scores (Fig. 1A) and component loadings (Fig. 1B), the four defined groups include unirradiated samples, but also samples irradiated with 1 and $10 \mathrm{kGy}$, making impossible to point out which parameter variations characterize better each of the studied groups $(0,1$ and $10 \mathrm{kGy}$ ). This result clearly indicates that, when considered from a global point of view, the changes resulting from irradiation treatment are not enough to separate each of the corresponding groups.

Nevertheless, gamma irradiation seemed to have caused changes in a species-dependent manner. In fact, the object scores corresponding to each plant species were clearly separated (Fig. 1C), especially for A. citrodora. The defined dimensions had, off course, the same Cronbach's $\alpha$ and eigenvalues, including also the same percentage of variance. By comparing Fig. $\mathbf{1 B}$ and $\mathbf{C}$, it is evident that the major differences in lemon verbena were caused on carbohydrates, physical parameters, malic acid, oxalic acid, total organic acids, C17:0, TBARS formation inhibition, reducing power and DPPH scavenging activity (all in methanolic extracts) and phenol content in infusions; on the other hand, energy, reducing sugars, C11:0, C22:0 and C20:3n3 + C21:0 suffer minor changes. The main differences on lemon balm were observed for protein, phenols (methanolic extracts) and reducing power (infusions), while ash, carbohydrates, C8:0, C13:0, C15:0, C16:0, SFA, and $\beta-$ carotene bleaching inhibition remain almost unchanged. Since the object scores of peppermint are in symmetric position in relation to lemon balm, the main characteristic alterations for peppermint are exactly the inverse to those verified in lemon balm. Lastly, the most sensitive parameters of bastard balm samples were C11:0, C14:0, C18:2n6 and DPPH scavenging activity (infusion), whereas fat, $\alpha$-tocopherol,

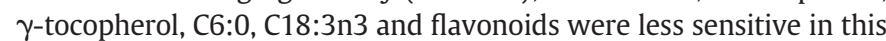
species.

\section{Conclusion}

When considered individually, the effects of gamma-irradiation (up to $10 \mathrm{kGy}$ ) in the chemical/nutritional and antioxidant properties of lemon verbena, lemon balm, bastard balm and peppermint proved to have statistical significance in particular cases. Nonetheless, when analyzed under an integrated approach, unirradiated and irradiated samples were grouped indiscriminately (as it might be deduced from the PCA plots), indicating that irradiation treatment did not cause sufficient changes to define a specific chemical profile. Interestingly, the way by which each species was affected by irradiation seemed to be characterized by some specificity, as revealed by the PCA plot of object scores. Overall, it might be considered that gamma irradiation treatment (up to $10 \mathrm{kGy}$ ) is a feasible conservation technology for the assayed Lamiaceae and Verbenaceae species. This is an interesting result because the $10 \mathrm{kGy}$ dose allows obtaining disinfested and decontaminated samples.

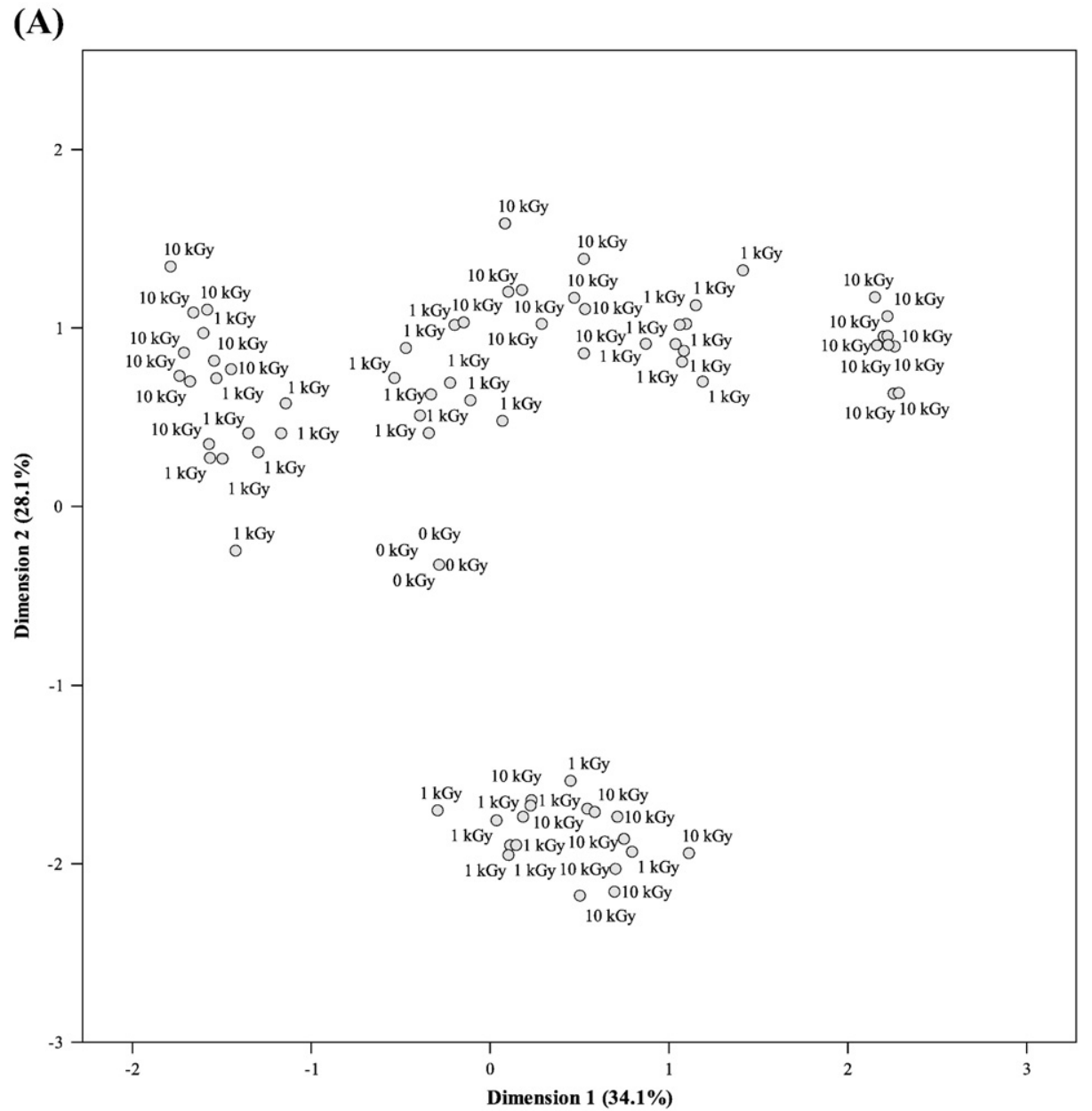

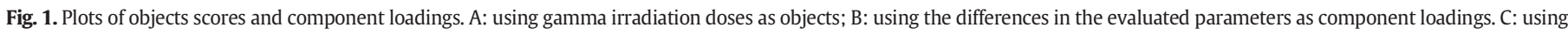
the assayed Lamiaceae and Verbenaceae species as objects. 
(B)

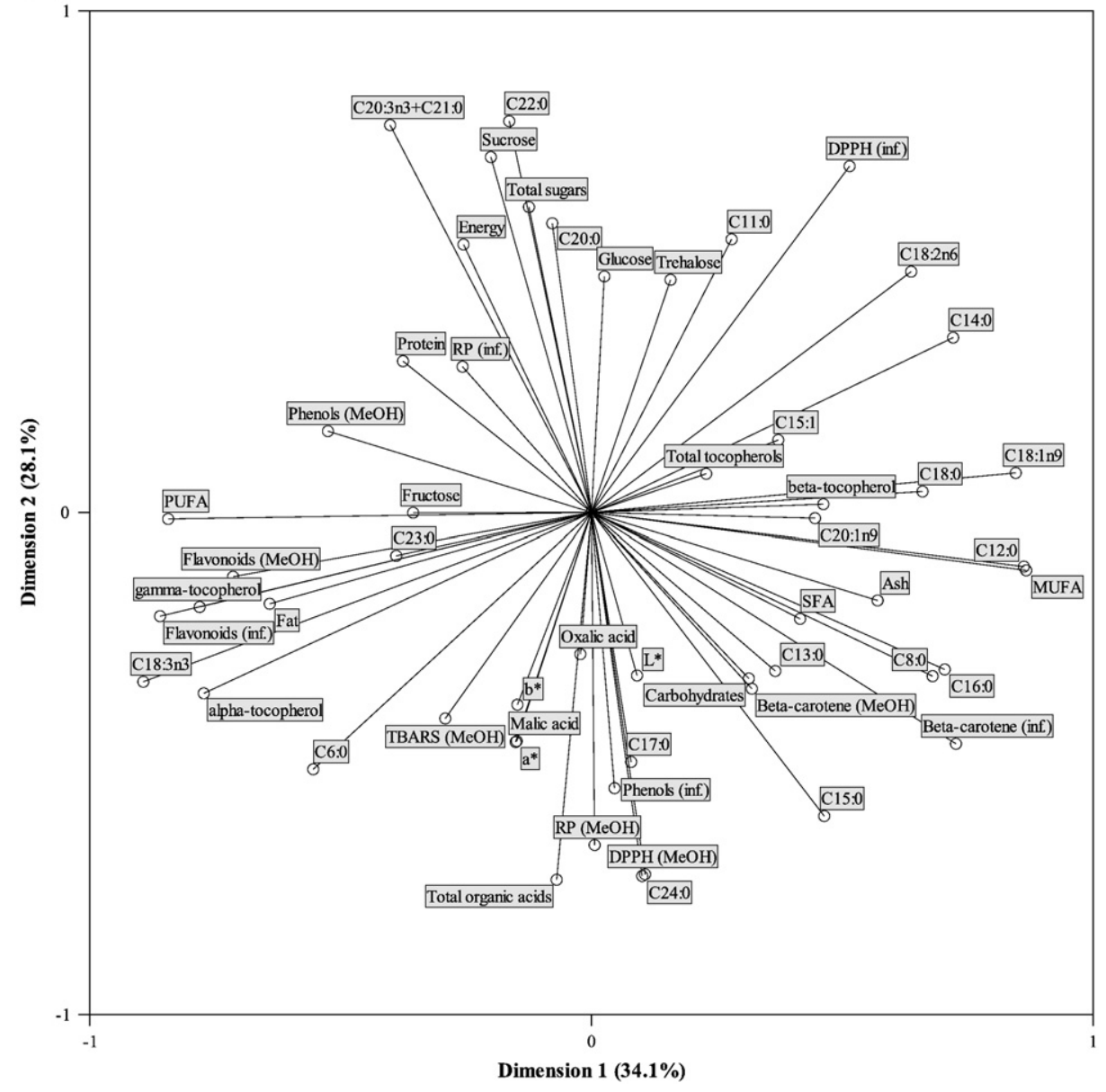

Fig. 1 (continued).

\section{Acknowledgments}

The authors are grateful to project PRODER no. 53514, AROMAP, for financial support of the work and E. Pereira grant, and to Fundação para a Ciência e a Tecnologia (FCT, Portugal) for financial support to CIMO (strategic project PEst-OE/AGR/UI0690/2011). L. Barros thanks "Compromisso para a Ciência 2008" for her contract. J.C.M. Barreira also thanks FCT, POPH-QREN and FSE for his grant (BPD/72802/2010). The authors are also grateful to "MaisErvasAromáticas e Medicinais".

Conflict of interest

The authors declare no conflict of interest.

\section{Appendix A. Supplementary data}

Supplementary data to this article can be found online at http://dx. doi.org/10.1016/j.foodres.2014.11.047.

\section{References}

AOAC (1995). Official methods of analysis. Association of Official Analytical Chemists, vol. 16, (Arlington VA, USA).

ASTM, American Society for Testing Materials (1992). Practice for using the Fricke reference standard dosimetry system, ASTM E1026. Annual book of ASTM standards, 12.02, Philadelphia, PA.

Barros, L., Pereira, E., Calhelha, R.C., Dueñas, M., Carvalho, A.M., Santos-Buelga, C., et al. (2013). Bioactivity and chemical characterization in hydrophilic and lipophilic compounds of Chenopodium ambrosioides L. Journal of Functional Foods, 5, $1732-1740$.

Byun, M.W., Kang, I.J., \& Mori, T. (1996). Effect of $\gamma$-irradiation on the water soluble components of soybeans. Radiation Physics and Chemistry, 47, 155-160.

Byun, M.W., Yook, H.S., Kim, K.S., \& Chung, C.K. (1999). Effects of gamma irradiation on physiological effectiveness of Korean medicinal herbs. Radiation Physics and Chemistry, 54, 291-300.

Byun, M.W., Yook, H.S., Kwon, O.J., \& Kang, I.J. (1997). Effect of gamma irradiation on physicochemical properties of Korean red ginseng powder. Radiation Physics and Chemistry, 49, 483-489.

Chmielewski, A.G., \& Migdał, W. (2005). Radiation decontamination of herbs and spices. Nukleonika, 50, 179-184.

Dastmalchi, K., Dorman, H.J.D., Oinonen, P.P., Darwis, Y., Laakso, I., \& Hiltunen, R. (2008). Chemical composition and in vitro antioxidative activity of a lemon balm (Melissa officinalis L.) extract. LWT-Food Science and Technology, 41, 391-400.

Dias, M.I., Barros, L., Sousa, M.J., \& Ferreira, I.C.F.R. (2012). Systematic comparison of nutraceuticals and antioxidant potential of cultivated, in vitro cultured and commercial Melissa officinalis samples. Food and Chemical Toxicology, 50, 1866-1873.

Fernandes, Â., Antonio, A.L. Barreira, J.C.M. Oliveira, M.B.P.P. Martins, A. \& Ferreira, I.C.F.R. (2012). Effects of gamma irradiation on physical parameters of Lactarius deliciosus wild edible mushrooms. Postharvest Biology and Technology, 74, 79-84.

Fernandes, Â., Barreira, J.C.M., Antonio, A.L., Santos, P.M.P., Martins, A., Oliveira, M.B.P.P. et al. (2013). Study of chemical changes and antioxidant activity variation induced by gamma-irradiation on wild mushrooms: Comparative study through principal component analysis. Food Research International, 54, 18-25.

Haleem, R.M., Salem, M.Y., Fatahallah, F.A., \& Abdelfattah, L.E. (2014). Quality in the pharmaceutical industry-A literature review. Saudi Pharmaceutical Journal, http://dx.doi.org/10.1016/j.jsps.2013.11.004.

Hsu, W. -Y., Simonne, A., Jitareerat, P., \& Marshall, M.R., Jr. (2010). Low-dose irradiation improves microbial quality and shelf life of fresh mint (Mentha piperita L.) without compromising visual quality. Journal of Food Science, 75, M222-M230.

Ibrahim, M.A., Mohammed, A., Isah, M.B., \& Aliyu, A.B. (2014). Anti-trypanosomal activity of African medicinal plants: A review update. Journal of Ethnopharmacology, 154, 26-54. 
(C)

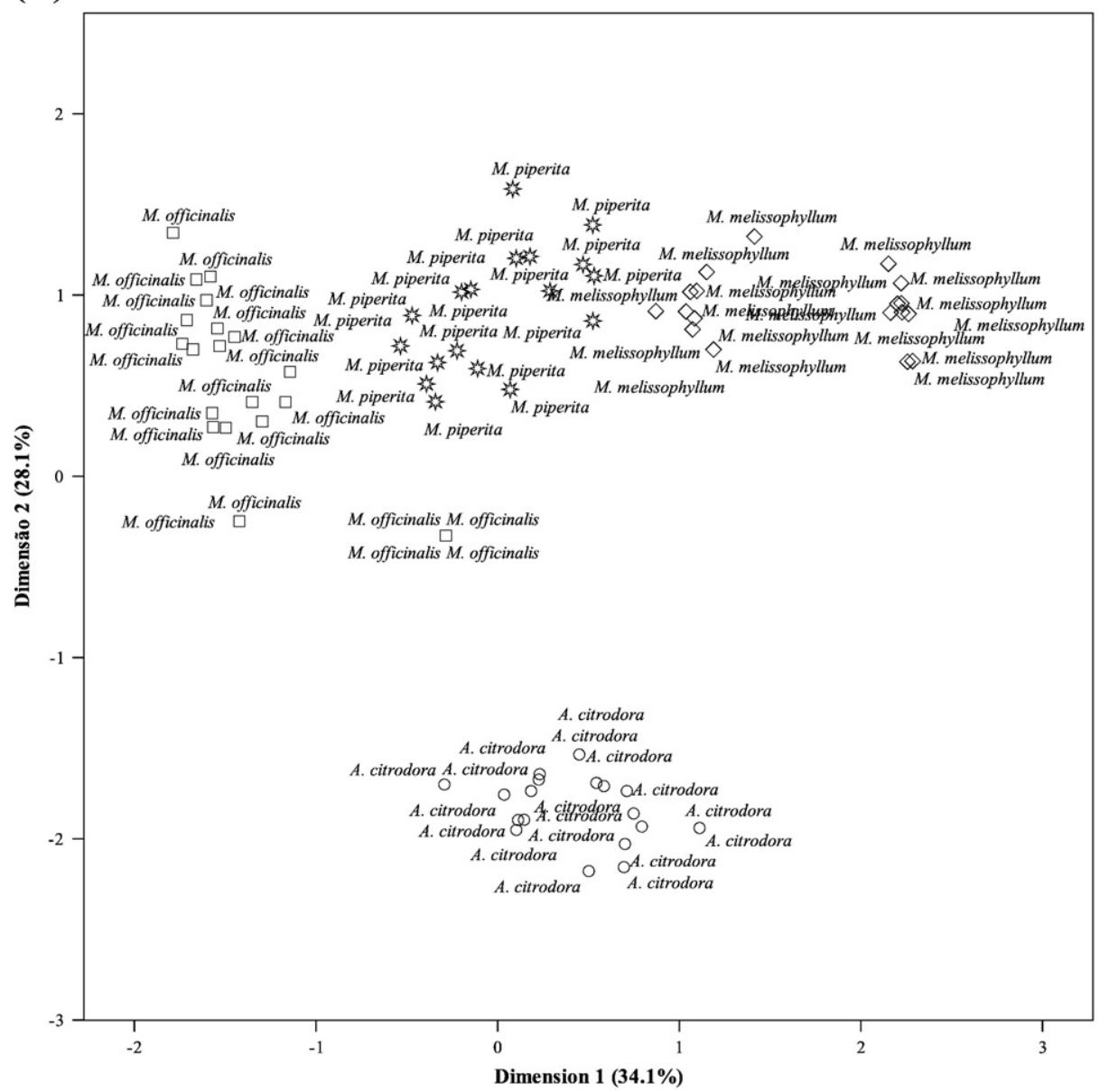

Fig. 1 (continued).

Jo, C., Son, J.H., Shin, M.G., \& Byun, M.W. (2003). Irradiation effects on color and functional properties persimmon (Diospyros kaki L. folium) leaf extract and licorice (Glycyrrhiza uralensis Fisher) root extract during storage. Radiation Physics and Chemistry, 67, $143-148$.

Kamdem, J.P., Adeniran, A., Boligon, A.A., Klimaczewski, C.V., Elekofehinti, O.O., Hassan, W., et al. (2013). Antioxidant activity, genotoxicity and cytotoxicity evaluation of lemon balm (Melissa officinalis L.) ethanolic extract: Its potential role in neuroprotection. Industrial Crops and Products, 51, 26-34.

Kapp, K., Hakala, E., Orav, A., Pohjala, L., Vuorela, P., Püssa, T., et al. (2013). Commercial peppermint (Mentha $\times$ piperita L.) teas: Antichlamydial effect and polyphenolic composition. Food Research International, 53, 758-766.

Kausar, T., Akram, K., \& Kwon, J. -H. (2013). Comparative effects of irradiation, fumigation, and storage on the free amino acids and sugar contents of green, black and oolong teas. Radiation Physics and Chemistry, 86, 96-101.

Migdal, W., \& Owczarczyk, B. (1998). The effect of ionizing radiation on microbiological decontamination of medical herbs and biologically active compounds. Radiation Physics and Chemistry, 52, 91-94.

Molins, R. (2001). Food irradiation. Principles and applications. USA: John Wiley \& Sons.

Nagy, T.O., Solar, S., Sontag, G., \& Koenig, J. (2011). Identification of phenolic components in dried spices and influence of irradiation. Food Chemistry, 128, 530-534.
Pereira, E., Barros, L., \& Ferreira, I.C.F.R. (2013). Chemical characterization of Ginkgo biloba L. and antioxidant properties of its extracts and dietary supplements. Industrial Crops and Products, 51, 244-248.

Ragone, M.I., Sella, M., Conforti, P., Volonté, M.G., \& Consolini, A.E. (2007). The spasmolytic effect of Aloysia citriodora, Palau (South American cedrón) is partially due to its vitexin but not isovitexin on rat duodenums. Journal of Ethnopharmacology, 113, 258-266.

Skrzypczak-Pietraszeka, E., \& Pietraszek, J. (2012). Chemical profile and seasonal variation of phenolic acid content in bastard balm (Melittis melissophyllum L., Lamiaceae). Journal of Pharmaceutical and Biomedical Analysis, 66, 154-161.

Small, E. (1996). Culinary herbs (2nd ed.). National Research Council Canada.

Taipina, M.S., Lamardo, L.C.A., Rodas, M.A.B., \& Mastro, N.L. (2009). The effects of gamma irradiation on the vitamin $\mathrm{E}$ content and sensory qualities of pecan nuts (Carya illinoensis). Radiation Physics and Chemistry, 78, 611-613.

Tissot, C., Grdanovska, S., Barkatt, A., Silverman, J., \& Al-Sheikhly, M. (2013). On the mechanisms of the radiation-induced degradation of cellulosic substances. Radiation Physics and Chemistry, 84, 185-190.

Warner, K., Miller, J., \& Demurin, Y. (2008). Oxidative stability of crude mid-oleic sunflower oils from seeds with $\gamma$ - and $\delta$-tocopherol levels. Journal of the American Chemical Society, 85, 529-533. 